\title{
Supporting Information: High In-Plane Thermal Conductivity of Aluminum Nitride Thin Films
}

Md Shafkat Bin Hoque, ${ }^{\dagger}$ Yee Rui Koh, ${ }^{\dagger}$ Jeffrey L. Braun, ${ }^{\dagger}$ Abdullah Mamun, ${ }^{\ddagger}$ Zeyu Liu, ${ }^{\mathbb{I l}}$ Kenny Huynh, ${ }^{\S}$ Michael E. Liao, ${ }^{\S}$ Kamal Hussain, ${ }^{\ddagger}$ Zhe Cheng, $\|$ Eric R. Hoglund, ${ }^{\perp}$ David H. Olson, ${ }^{\dagger}$

John A. Tomko, ${ }^{\perp}$ Kiumars Aryana, ${ }^{\dagger}$ Roisul Galib, ${ }^{\dagger}$ John T. Gaskins, ${ }^{\dagger}$ Mirza Mohammad Mahbube Elahi," Zayd C. Leseman, ${ }^{\circledR, \triangle}$ James M. Howe, ${ }^{\perp}$ Tengfei Luo, II Samuel Graham, $\|, \nabla$ Mark S. Goorsky, ${ }^{\S}$ Asif Khan, ${ }^{\ddagger}$ and Patrick E. Hopkins $*, \dagger, \perp, \dagger$

$\dagger$ Department of Mechanical and Aerospace Engineering, University of Virginia, Charlottesville, Virginia 22904, USA $\ddagger$ Department of Electrical Engineering, University of South Carolina, Columbia, South Carolina 29208, USA IDepartment of Aerospace and Mechanical Engineering, University of Notre Dame, Notre Dame, Indiana 46556, USA

§Department of Materials Science and Engineering, University of California, Los Angeles, California 90095, USA \|George W. Woodruff School of Mechanical Engineering, Georgia Institute of Technology, Atlanta, Georgia 30332, USA

$\perp$ Department of Materials Science and Engineering, University of Virginia, Charlottesville, Virginia 22904, USA \#Department of Electrical and Computer Engineering, University of New Mexico, Albuquerque, New Mexico 87131, USA

@ Department of Mechanical Engineering, King Fahd University of Petroleum \& Minerals, Dhahran, Eastern Province 31261, Saudi Arabia

$\triangle$ Interdisciplinary Research Center for Advanced Materials, King Fahd University of Petroleum \& Minerals, Dhahran, Eastern Province 31261, Saudi Arabia

$\nabla S c h o o l$ of Materials Science and Engineering, Georgia Institute of Technology, Atlanta, Georgia 30332, USA $\dagger \dagger$ Department of Physics, University of Virginia, Charlottesville, Virginia 22904, USA

E-mail: phopkins@virginia.edu 


\section{Contents}

Section S1. Growth details of the AlN thin films (Page 3)

Section S2. Characterizations of point- and line-defect concentrations in the AlN thin films (Page 3)

Section S3. Scanning transmission electron microscopy (STEM) characterization of the AlN thin films (Page 5)

Section S4. Analytical expression of steady-state temperature rise (Page 6)

Section S5. Sensitivity analysis of SSTR measurements (Page 7)

Section S6. Steady-state temperature rise profiles in the AlN thin films (Page 11)

Section S7. Details of the SSTR measurement and fitting procedure (Page 14)

Section S8. SSTR measurements of thin film and anisotropic bulk control samples (Page 17)

Section S9. SSTR measurements of the single crystal AlN layer (Page 18)

Section S10. Influence of interface on SSTR measurements (Page 19)

Section S11. TDTR measurements of the $3.05 \mu \mathrm{m}$ AlN thin film (Page 20)

Section S12. TDTR measurements of Si at low temperatures (Page 22)

Section S13. Uncertainty analysis of TDTR and SSTR measurements (Page 23)

Section S14. Temperature-dependent thermal conductivity of thin film materials (Page 25)

Section S15. Boltzmann transport equation (BTE) method used to calculate the AlN meanfree-path spectra (Page 26)

Section S16. A simplified thermal resistance model for the $3.05 \mu \mathrm{m}$ AlN film (Page 27)

Table S1. Parameters used in the measurements of room-temperature in-plane thermal conductivities of the AlN thin films (Page 30)

Table S2. Bulk in-plane thermal conductivities of the thin film materials presented in Figure 3b (Page 31) 


\section{S1. Growth details of the AIN thin films}

A custom metal-organic chemical vapor deposition (MOCVD) reactor facilitated with a fast precursor switching manifold is used to grow the 3.05, 3.75, and $6 \mu \mathrm{m}$ AlN thin films. $0.2^{\circ}$ mis-cut basal plane sapphire wafers of two-inch diameters are utilized as the substrate material for the film growth. A pulsed epitaxy sequence initiates the sample growth at $1100{ }^{\circ} \mathrm{C}$ under the presence of $\mathrm{Al}$ and $\mathrm{N}$ precursors (trimethyl aluminum and $\mathrm{NH}_{3}$, respectively). By controlling the ratio and flow rate of trimethyl aluminum and $\mathrm{NH}_{3}$ into the growth chamber, an air-pocketed AlN layer is created at the AlN/sapphire interface. The presence of this layer helps manage the strain and prevents cracking as the thickness of the AIN film increases. Subsequent adjustment of the flow rate and increase of chamber temperature to $1200{ }^{\circ} \mathrm{C}$ result in smooth AIN layers. Thin films of different thicknesses are grown by modulating the time of the sample growth. The purity of the $\mathrm{Al}$ and $\mathrm{N}$ precursors along with the high temperatures of the growth chamber result in low concentrations of point defects in our AlN thin films.

\section{S2. Characterizations of point- and line-defect concentrations in the AIN thin films}

The AlN thin films consist of a top single crystal layer and a bottom nucleation layer. The top single crystal layer has very low concentrations of point and line defects, whereas the bottom nucleation layer has higher concentrations of these defects. Secondary ion mass spectrometry (SIMS) is used to measure the point-defect concentrations in the AlN films. The O, Si, and C impurity concentrations in the single crystal layer are on the order of $\sim 10^{15}$ to $10^{17}$ atoms cm ${ }^{-3}$. $^{1}$ The impurity concentrations of the commercial grade AIN wafers can be several orders of magnitude higher compared to the single crystal layer of our AlN films. For example, the O, Si, and $\mathrm{C}$ concentrations in the $500 \mu \mathrm{m}$ commercial AlN wafer presented in Figure 4a are on the order of $\sim 10^{18}$ to $10^{19}$ atoms $\mathrm{cm}^{-3} .{ }^{1}$ The bulk, high-purity, single crystal AIN measured by Slack et al. ${ }^{2}$ (shown in Figure 4a) also has an oxygen impurity concentration of $(42 \pm 2) \times 10^{18}$ atoms cm $^{-3}$. 
The dislocation density is calculated by determining the number of dislocations per unit area from the cross section two beam condition images. The dislocation density is on the order of $10^{8} \mathrm{~cm}^{-2}$ in the single crystal layer and one to two orders of magnitude higher in the nucleation layer. The dislocations are oriented along the $c$-axis in both layers of the films. ${ }^{1,3}$ As shown in the Supplementary Information of Sun et al. ${ }^{4}$ dislocations can reduce GaN thermal conductivity when the dislocation density is higher than $10^{8} \mathrm{~cm}^{-2}$. As both AlN and GaN are III-V compound semiconductors and have a wurtzite crystal structure,${ }^{5}$ it is reasonable to expect that the effects of dislocations on the thermal conductivity of the two materials are nearly the same. ${ }^{6-8}$ Therefore, a similar assumption can be made regarding the threshold dislocation density necessary to reduce AlN thermal conductivity. Under this assumption, the thermal conductivity reduction due to dislocations is minimal in the single crystal layer of our AlN thin films. However, the nucleation layer has a more pronounced thermal conductivity reduction induced by dislocations.

The single crystal layer has lower impurity concentrations than the commercially available previously studied AlN. As a result, the thermal conductivity of this single crystal layer is very high, $\sim 320 \mathrm{~W} \mathrm{~m}^{-1} \mathrm{~K}^{-1}$ as shown by Koh et al. ${ }^{3}$ The nucleation layer, on the other hand, has higher concentrations of point and line defects and a lower thermal conductivity. As SSTR probes through both single crystal and nucleation layers, the AlN thin films have an in-plane thermal conductivity of $\sim 260 \pm 40 \mathrm{~W} \mathrm{~m}^{-1} \mathrm{~K}^{-1}$. This in-plane thermal conductivity is still close to Slack et al.'s ${ }^{2}$ prediction of bulk, defect-free, single crystal AlN thermal conductivity of $\sim 319 \mathrm{~W} \mathrm{~m}^{-1}$ $\mathrm{K}^{-1}$. This proves the high quality of our AlN thin films. 


\section{S3. Scanning transmission electron microscopy (STEM) characterization of the AIN thin films}

Figure S1 shows the scanning transmission electron microscopy (STEM) image of a $\sim 3.3 \mu \mathrm{m}$ AlN thin film grown in the same way as the AlN films used in this study. Additional STEM characterizations of the AlN films can be found in Koh et al. ${ }^{3}$ The AlN films have two distinct layers: a nucleation layer near the AlN/sapphire interface, followed by a single crystal layer. The thickness of this nucleation layer increases from $\sim 600 \mathrm{~nm}$ to $1.5 \mu \mathrm{m}$ as the AlN film thickness increases from $\sim 3$ to $22 \mu \mathrm{m}$, respectively. ${ }^{3}$ This demonstrates that with the increase of film thickness, nucleation layer occupies a smaller percentage of the total thickness. The presence of this nucleation layer helps manage the strain and prevents cracking during the growth of thick single crystal layer.

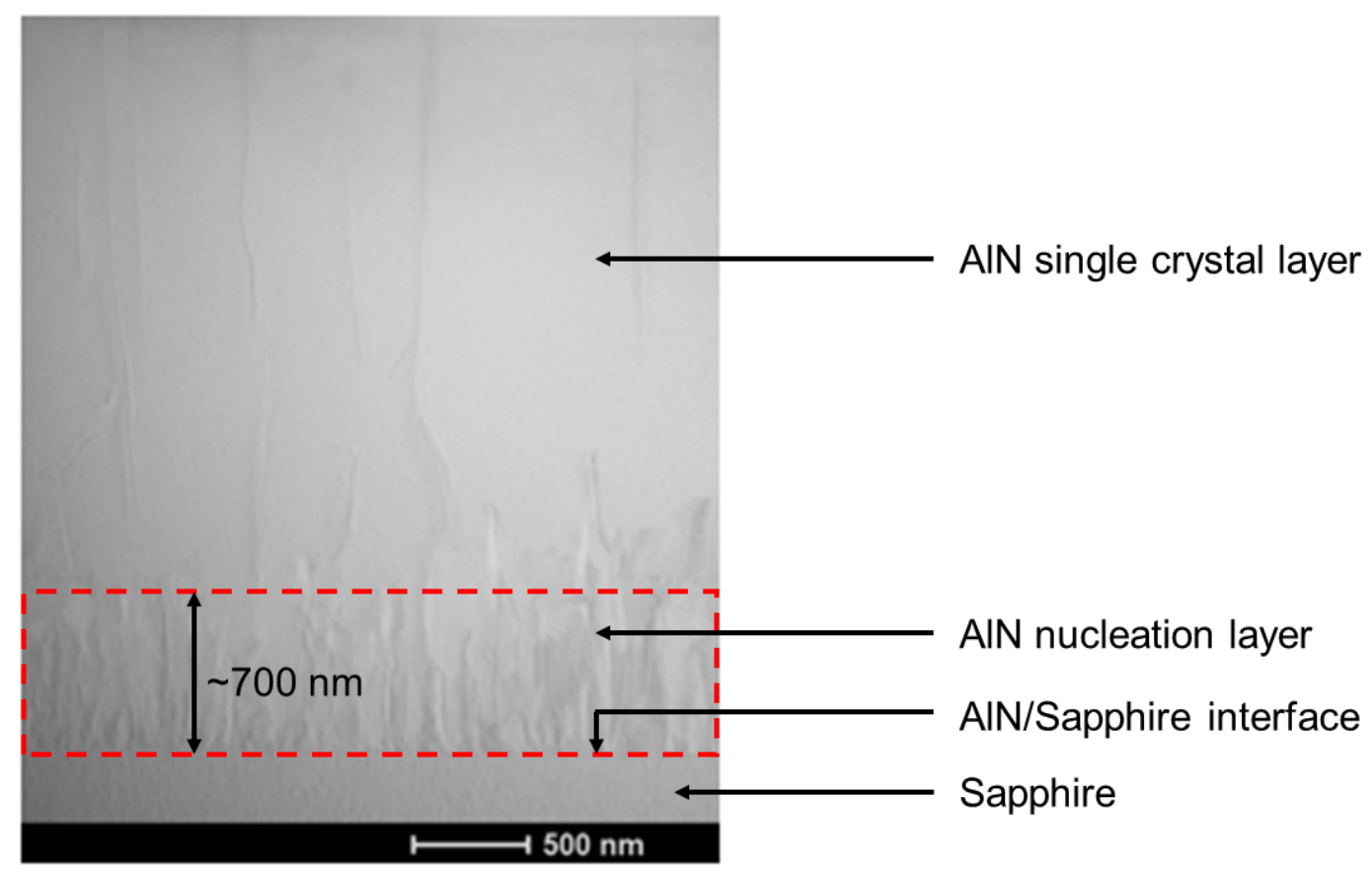

Figure S1: STEM image of a $\sim 3.3 \mu \mathrm{m}$ AlN thin film showing the single crystal and nucleation layers. 


\section{S4. Analytical expression of steady-state temperature rise}

In optical pump-probe measurements, the temperature rise at the sample surface is proportionally captured by the probe reflectivity change. Assuming radial symmetry in the cylindrical coordinate, the probe-averaged temperature rise $\left(T_{P}\right)$ for a bulk, single-layered material can be expressed as the following ${ }^{9-11}$

$$
T_{P}=\frac{4}{r_{1}^{2}} \int_{0}^{\infty} T(r) \exp \left(\frac{-2 r^{2}}{r_{1}^{2}}\right) r d r
$$

Here, $r$ represents radial coordinate, and $T(r)$ is the distribution of temperature oscillations at the sample surface due to the modulated heating event from the pump beam. Equation (S1) assumes that both pump and probe beams have Gaussian profiles and negligible optical penetration depths. The $1 / \mathrm{e}^{2}$ pump and probe radii are denoted by $r_{0}$ and $r_{1}$, respectively.

In the extreme limits of low modulation frequency such as an unmodulated CW laser source, equation (S1) can be analytically solved to become ${ }^{9,11}$

$$
T_{P}=\frac{P_{0}}{\sqrt{2 \pi\left(r_{0}^{2}+r_{1}^{2}\right)(k \|)(k \perp)}}=\frac{P_{0}}{r_{01} \sqrt{2 \pi(k \|)(k \perp)}}
$$

Here, $r_{01}=\sqrt{r_{0}^{2}+r_{1}^{2}}$ is the effective radius, and $P_{0}$ is average power absorbed by the sample. For a bulk material, equation (S2) provides an approximation of the steady-state temperature rise for conditions similar to SSTR measurement settings. As shown here, the probe-averaged steady-state temperature rise depends on the geometric mean of in-plane and cross-plane thermal conductivities. By rearranging the terms of equation (S2), an expression for $\sqrt{(k \|)(k \perp)}$ can be obtained.

For layered structures such as thin films on a substrate, analytically obtaining expressions similar to equation (S2) becomes challenging. For such cases, the radially symmetric heat diffusion 
equation is numerically solved to determine the thermal conductivity tensor of SSTR measurements. ${ }^{10,12}$

\section{S5. Sensitivity analysis of SSTR measurements}

The SSTR sensitivity expression can by obtained by following a methodology similar to that of Yang et al. ${ }^{13}$ For FDTR measurements, Yang et al. ${ }^{13}$ quantified the sensitivity, $S_{x}$, of the thermal model as the change in the phase $(\phi)$ signal caused by the variation of a parameter, $x$, within a tolerance limit of $\pm 10 \%$. According to this definition, $S_{x}=\left.\phi\right|_{1.1 x}(\omega)-\left.\phi\right|_{0.9 x}(\omega)$, where $\omega$ is the frequency.

We adopt a similar approach for defining the sensitivity of SSTR measurements. However, since SSTR measures the magnitude ( $\propto$ steady-state temperature rise) instead of phase, a division term is added to the definition to make the comparison of sensitivities among samples of different properties and geometries fair. ${ }^{12}$ Therefore,

$$
S_{x}=\frac{\left|\triangle T_{1.1 x}\left(r_{01}\right)-\triangle T_{0.9 x}\left(r_{01}\right)\right|}{\triangle T_{x}\left(r_{01}\right)}
$$

Here, $\triangle T_{x}$ is the temperature rise predicted by the steady-state thermal model ${ }^{12}$ for an input parameter $x$. We define $S_{x}$ in terms of effective radius, $r_{01}$, as SSTR offers a convenient way to change sensitivities to different parameters by using multiple pump and probe radii. However, $S_{x}$ can also be expressed in terms of modulation frequency or any other parameters of interest.

As discussed in detail by Braun et al., ${ }^{12}$ SSTR measures the geometric mean of $k \|$ and $k \perp$ for bulk materials, and has nearly equal sensitivity to both $k \|$ and $k \perp$. However, for thin film measurements, this changes based on thin film to substrate thermal conductivity ratios: ${ }^{12}$

(a) If the thin film thermal conductivity is much higher than that of the substrate, heat flows 
predominantly along the in-plane direction of the film. As a result, the temperature gradient along the in-plane direction is much more pronounced compared to cross-plane. In this case, the in-plane thermal conductivity of the film dominates the sensitivity calculations allowing SSTR to directly

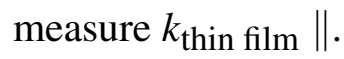

(b) When the thermal conductivity of the thin film and substrate are equal, heat flows almost equally along the in-plane and cross-plane directions. This results in near-identical temperature gradients in both directions. For such a case, sensitivity to the in-plane and cross-plane thermal conductivities of the film are nearly the same. Thus, SSTR measures $\sqrt{\left(k_{\text {thin film }} \|\right)\left(k_{\text {thin }} \text { film } \perp\right)}$.

(c) For the case where the thin film thermal conductivity is much lower than substrate, the majority of the heat flows towards the highly conductive substrate. Here, the temperature gradient in the cross-plane direction becomes very large. As a result, sensitivity to the cross-plane thermal conductivity of the thin film is much higher compared to that of in-plane. Therefore, SSTR measurements represent $k_{\text {thin film }} \perp$.

To determine a priori whether SSTR measurements represent the in-plane or cross-plane thermal conductivity of the AIN thin films, we depend on sensitivity calculations. For this purpose, we conservatively estimate that the cross-plane thermal conductivities of the AlN films lie between 150 and $370 \mathrm{~W} \mathrm{~m}^{-1} \mathrm{~K}^{-1}$ at room temperature. The vast majority of the literature reported crossplane thermal conductivities of single crystal AlN fall within this range. ${ }^{1-3,6,14,15}$ As the thin films used in this study are of high quality, ${ }^{1,3}$ it is reasonable to assume that the cross-plane thermal conductivities of our AlN films can also be found within these limits.

In Figure S2, the sensitivity, $S_{x}$, as a function of $k_{\mathrm{AlN}} \|$ is presented for cross-plane thermal conductivities of 370 and $150 \mathrm{~W} \mathrm{~m}^{-1} \mathrm{~K}^{-1}$. These calculations are performed by numerically solving equation (S3) in MATLAB. The values presented in Table S1 are used as input parameters. In addition, the $1 / \mathrm{e}^{2}$ pump and probe radii are taken as $10 \mu \mathrm{m}$. From Figure $\mathrm{S} 2$, it is evident that for all three thin films, there is negligible sensitivity to the cross-plane thermal conductivity 
of the AlN film. The sensitivity calculations are dominated by the in-plane thermal conductivity of the AlN thin film, regardless of what this thermal conductivity is. This indicates that SSTR measures the in-plane thermal conductivities of the AIN thin films. Figure S2 also shows that sensitivity to the substrate thermal conductivity keeps decreasing as the film thickness increases. SSTR measurements are not sensitive to the AIN/sapphire thermal boundary conductance.

We have verified our assumption regarding the cross-plane thermal conductivity limits by measuring the $3.05 \mu \mathrm{m}$ AIN film by TDTR (shown in Figure 5a). The measured cross-plane thermal conductivity is well within the limits presented here. Running similar sensitivity calculations at different temperatures for a wide range of cross-plane thermal conductivities also reveal the dominance of in-plane thermal conductivity. Thus, we can confidently say all SSTR measurements of the AlN thin films in this study represent the in-plane thermal conductivity. 

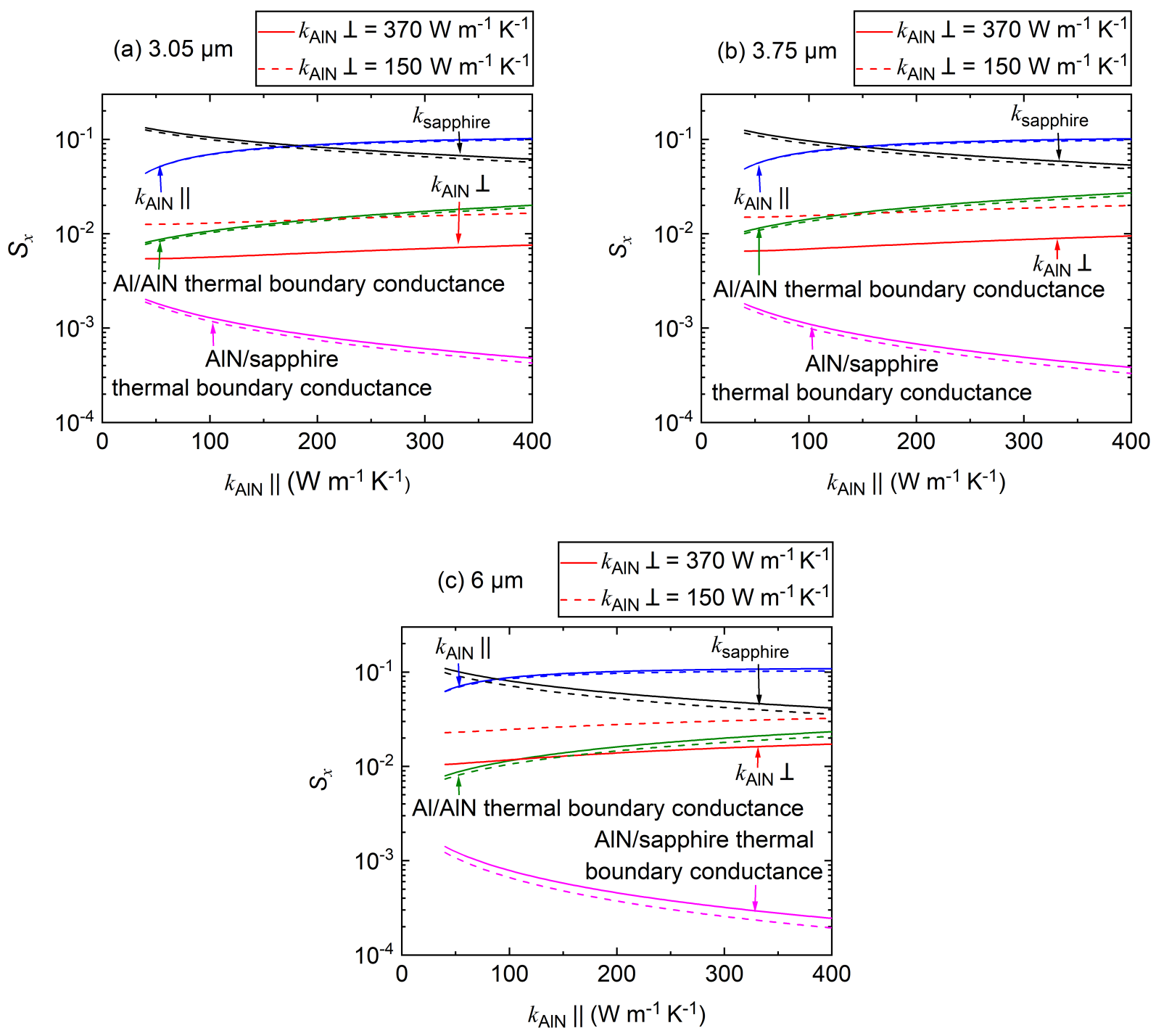

Figure S2: Sensitivity, $S_{x}$, to different parameters as a function of $k_{\text {AlN }} \|$ for (a) 3.05, (b) 3.75, and (c) $6 \mu \mathrm{m}$ AlN thin films. For these calculations, a modulation frequency of $100 \mathrm{~Hz}$ and coaxially focused $1 / \mathrm{e}^{2}$ pump and probe radii of $10 \mu \mathrm{m}$ are used. The solid and dashed lines correspond to the cross-plane thermal conductivities of 370 and $150 \mathrm{~W} \mathrm{~m}^{-1} \mathrm{~K}^{-1}$, respectively. 


\section{S6. Steady-state temperature rise profiles in the AIN thin films:}

The steady-state temperature rise profiles of SSTR measurements can be obtained by solving the cylindrical heat diffusion equation assuming radial symmetry and the presence of a heating source at the surface

$$
(k \|)\left\{\frac{1}{r} \frac{\partial T(z, r, t)}{\partial r}+\frac{\partial^{2} T(z, r, t)}{\partial r^{2}}\right\}+(k \perp) \frac{\partial^{2} T(z, r, t)}{\partial z^{2}}=C_{V} \frac{\partial T(z, r, t)}{\partial t}
$$

Here, $C_{V}, t, r$, and $z$ represent the volumetric heat capacity, time, distance along the in-plane, and cross-plane directions, respectively.

When the top surface of the material is in contact with an insulating medium (e.g., air), the boundary conditions can be written as the followings

$$
\begin{gathered}
T(0, r, t)=T_{\text {top }}(r, t) \\
\left.\left.\frac{\partial T(z, r, t)}{\partial r}\right|_{z=0}=-\frac{1}{(k \perp}\right) Q_{t o p}(r, t)
\end{gathered}
$$

Here, $T_{t o p}(r, t)$ and $Q_{t o p}(r, t)$ denote the temperature and heat flux at the top surface of the material under consideration, respectively. The initial condition $T(z, r, 0)=0$ describes the reference temperature with respect to which the steady-state temperature rise is calculated.

Using these boundary and initial conditions, equation (S4) can be solved for SSTR measurement settings such as CW laser sources and near surface energy absorption. A detailed description of this methodology was provided by Braun and Hopkins. ${ }^{10}$ We use the same methodology to numerically model the steady-state temperature rise profiles in the AlN thin films.

For these models, we assume that the limits estimated in Section S5 for cross-plane thermal conductivity of the AlN thin films are also applicable for in-plane. The assumption regarding the 
lower limit $\left(150 \mathrm{~W} \mathrm{~m}^{-1} \mathrm{~K}^{-1}\right)$ can be justified by the fact that these AlN films do not contain columnar grains or significant concentrations of dislocation line-defects. Therefore, the in-plane thermal conductivity is not expected to be lower than cross-plane for these high-quality AlN films ${ }^{16-18}$ contrary to the other group III nitride films reported in literature. ${ }^{4,19}$ In addition, our estimated upper limit $\left(370 \mathrm{~W} \mathrm{~m}^{-1} \mathrm{~K}^{-1}\right)$ is already higher than theoretical predictions of defect-free, single crystal AlN thermal conductivity. ${ }^{2,5}$

Figure $\mathrm{S} 3$ shows the steady-state temperature rise profiles of the AlN thin films corresponding to the estimated lower and upper bounds. MATLAB is used to generate these models. Here, it is evident that for all cases, the temperature gradient is much more pronounced along the in-plane direction compared to cross-plane. This scenario occurs as the majority of the heat flows along the in-plane direction of the AlN films. From these temperature profiles, it can be concluded that SSTR measurements represent the in-plane thermal conductivity of the AlN thin films. This conclusion remains unchanged for any combinations of in-plane and cross-plane thermal conductivities within these bounds. 

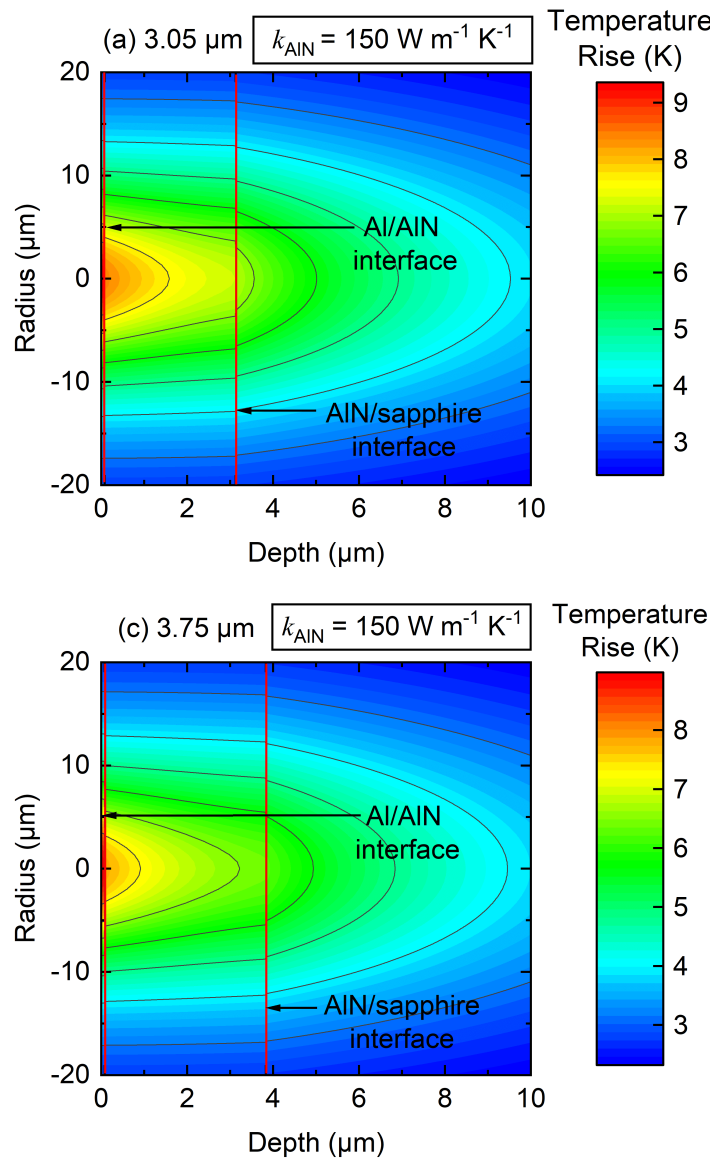

Temperature Rise (K)

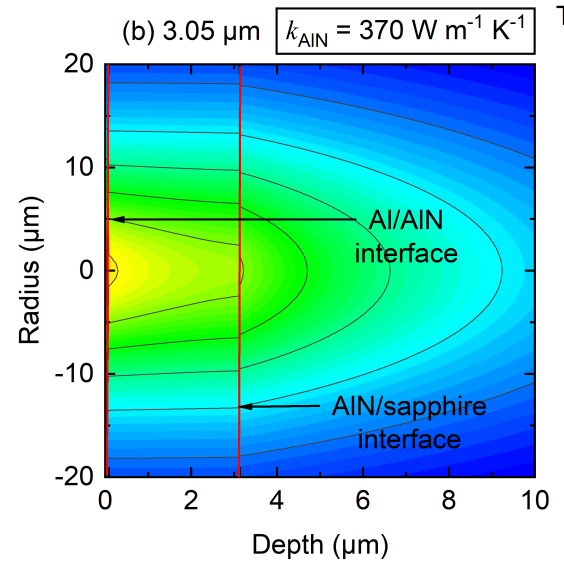

Temperature
Rise $(\mathrm{K})$
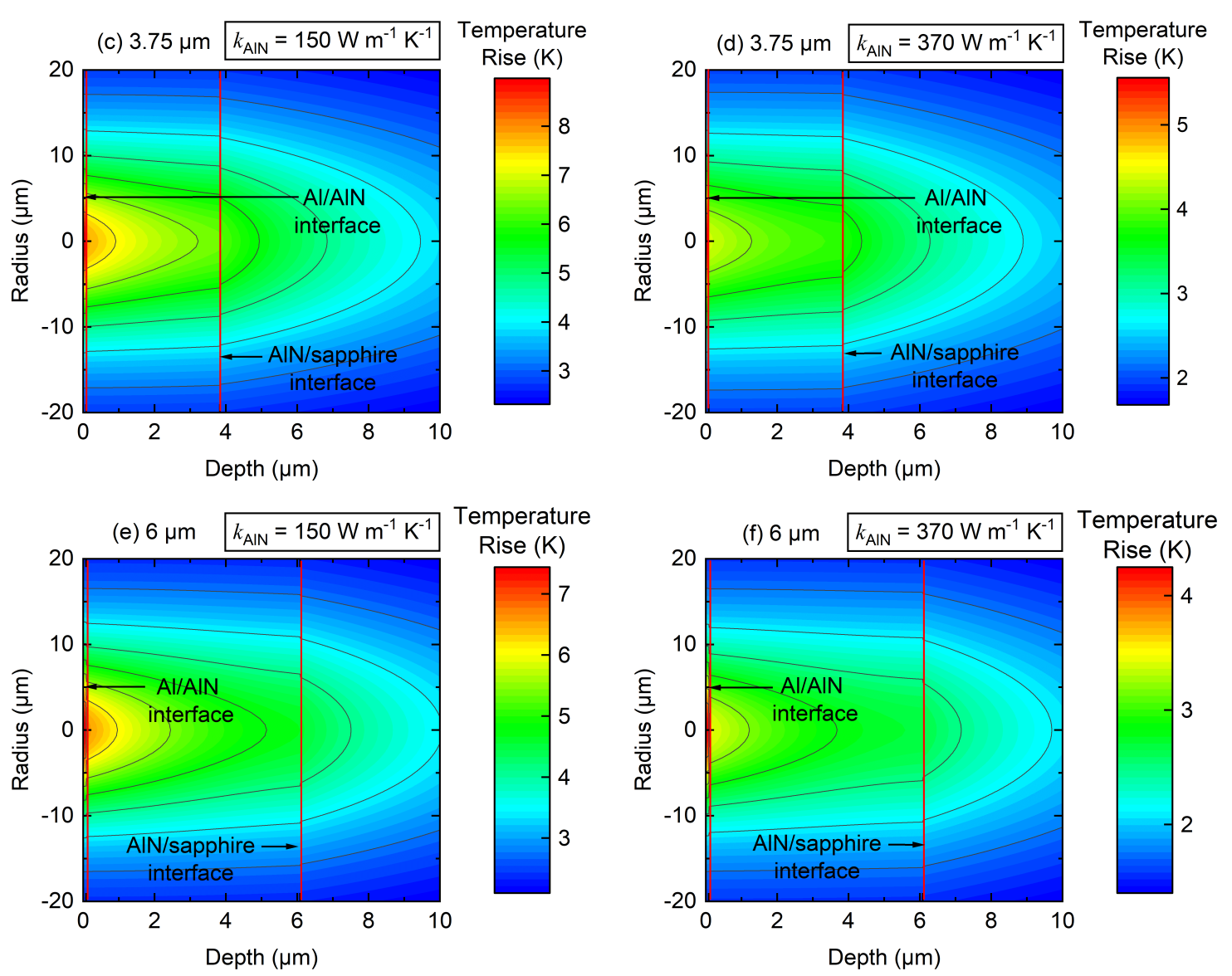

Figure S3: Steady-state temperature rise profiles in [(a) and (b)] 3.05, [(c) and (d)] 3.75, and [(e) and (f)] $6 \mu \mathrm{m}$ AlN films. For these calculations, a modulation frequency of $100 \mathrm{~Hz}$, pump power of $200 \mathrm{~mW}$, and coaxially focused $1 / \mathrm{e}^{2}$ pump and probe radii of $10 \mu \mathrm{m}$ are used. Panels (a), (c), and (e) correspond to $k_{\text {AIN }} \|=k_{\text {AlN }} \perp=150 \mathrm{~W} \mathrm{~m}^{-1} \mathrm{~K}^{-1}$, whereas for panels (b), (d), and (f), $k_{\text {AIN }} \|$ $=k_{\mathrm{AlN}} \perp=370 \mathrm{~W} \mathrm{~m}^{-1} \mathrm{~K}^{-1}$. The red lines represent the Al/AlN and AlN/sapphire interfaces. 


\section{S7. Details of the SSTR measurement and fitting procedure:}

For SSTR measurements, we fit for thermal conductivity, while other parameters are independently determined. The $\mathrm{Al} / \mathrm{sapphire,} \mathrm{Al} / \mathrm{Si}$, and $\mathrm{Al} / \mathrm{AlN}$ thermal boundary conductances are measured by TDTR. In Figure S4, we have presented $(\triangle V / V)$ vs $\triangle P$ for the room-temperature thermal conductivities of bulk sapphire, bulk Si, 3.05, 3.75, and $6 \mu \mathrm{m}$ AlN thin films. The material properties used to determine the in-plane thermal conductivities of the AlN thin films by SSTR at room temperature are tabulated in Table S1.

TDTR is used to measure the temperature-dependent thermal conductivities of reference sapphire. These sapphire thermal conductivities are used to determine the $\gamma$ values. At each temperature, $\gamma$ is calculated by comparing the experimentally measured $\triangle V /(V \triangle P)$ of sapphire reference to the $\triangle T$ predicted by the steady-state thermal model. These $\gamma$ values are then used to determine the thermal conductivities of bulk Si and AlN thin films by SSTR. In Figure S5, we have plotted the TDTR-measured sapphire values used to determine the in-plane thermal conductivities of the $3.05 \mu \mathrm{m}$ AlN film by SSTR. Our TDTR-measured sapphire thermal conductivities are in excellent agreement with literature. ${ }^{20}$

In our SSTR setup, pump and probe spot sizes are nearly equal. The room-temperature in-plane thermal conductivity of each AIN thin film is measured with two different objectives: $5 \mathrm{X}$ and $10 \mathrm{X}$ with corresponding $1 / \mathrm{e}^{2}$ pump radii of $\sim 20$ and $10 \mu \mathrm{m}$, respectively. The values measured by the two objectives are within $\sim 5 \%$ of each other. For the temperature-dependent measurements in this study, a 10X objective is used with one exception. Below $120 \mathrm{~K}, 10 \mathrm{X}$ measurements are highly influenced by the Al/AIN thermal boundary conductances and do not have enough sensitivity to the in-plane thermal conductivities of the AIN thin films. Therefore, to measure the in-plane thermal conductivity of the $3.05 \mu \mathrm{m}$ AlN film at $105 \mathrm{~K}$, a $5 \mathrm{X}$ objective is used. The thermal conductivities of bulk sapphire and Si are analyzed with a two layer model: Al transducer/sapphire or Si wafer. The thermal conductivities of the AlN films are analyzed with a three layer model: $\mathrm{Al}$ transducer/AlN thin film/sapphire substrate. 
For the bulk sapphire reference and Si calibration, SSTR-measured thermal conductivities represent $\sqrt{(k \|)(k \perp)}$. All TDTR measurements in this study represent the cross-plane thermal conductivity. For sapphire and Si, TDTR measures $k \perp$, whereas SSTR measures $\sqrt{(k \|)(k \perp)}$. Si has isotropic thermal conductivity ${ }^{21,22}$ while sapphire is weakly anisotropic. ${ }^{20,22,23}$ Therefore, comparisons between the TDTR- and SSTR-measured thermal conductivities of bulk sapphire or Si are quantitatively valid. On the other hand, for the AlN thin films, TDTR measures $k \perp$ and SSTR measures $k \|$. Thus, all SSTR measurements of the AlN thin films are described as the in-plane thermal conductivity, whereas all TDTR measurements of the AlN films are designated as the cross-plane thermal conductivity.

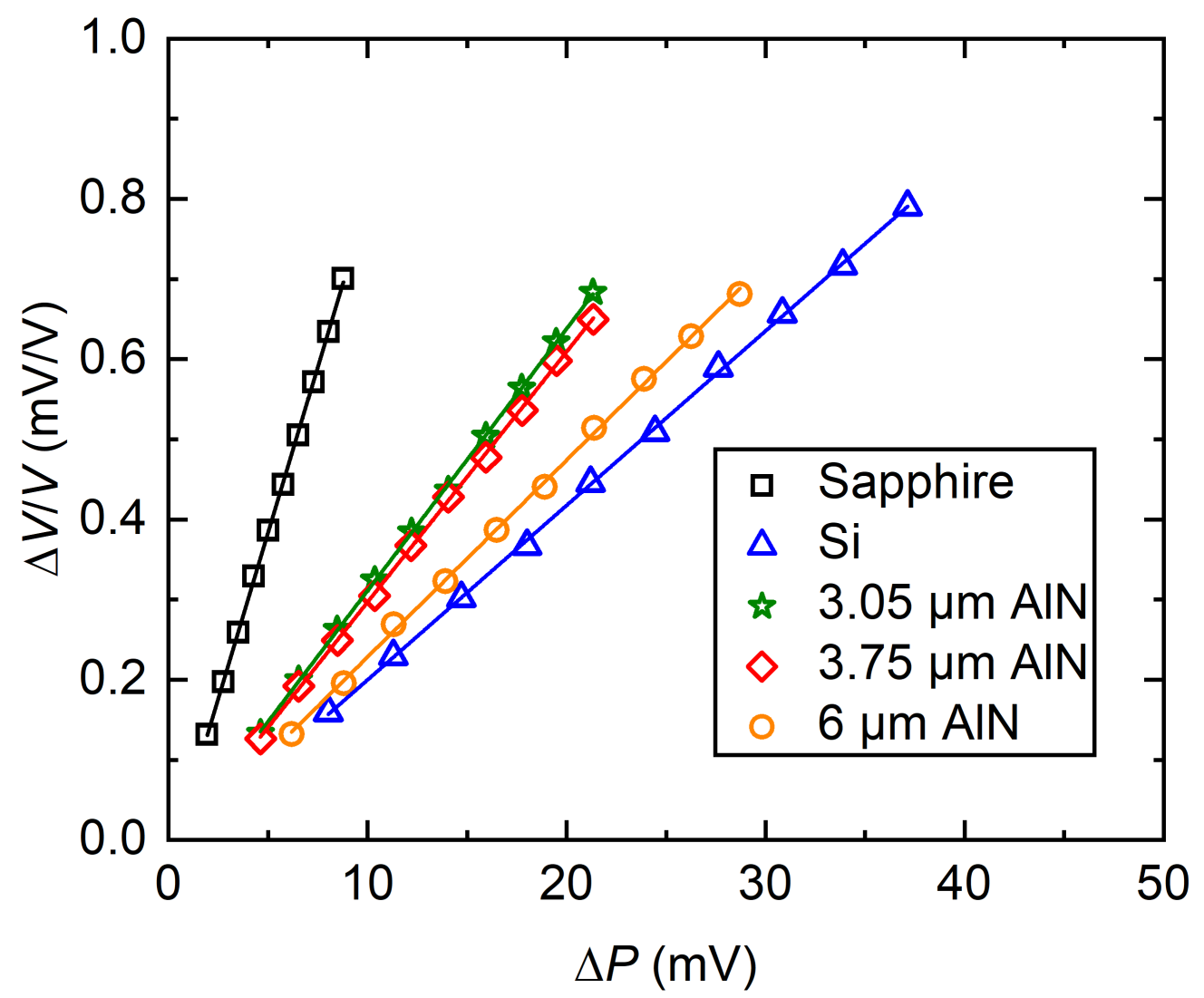

Figure S4: $(\triangle V / V)$ vs $\triangle P$ used in SSTR fitting for the thermal conductivities of bulk sapphire, bulk Si, 3.05, 3.75, and $6 \mu \mathrm{m}$ AlN thin films with a 10X objective at room temperature. 


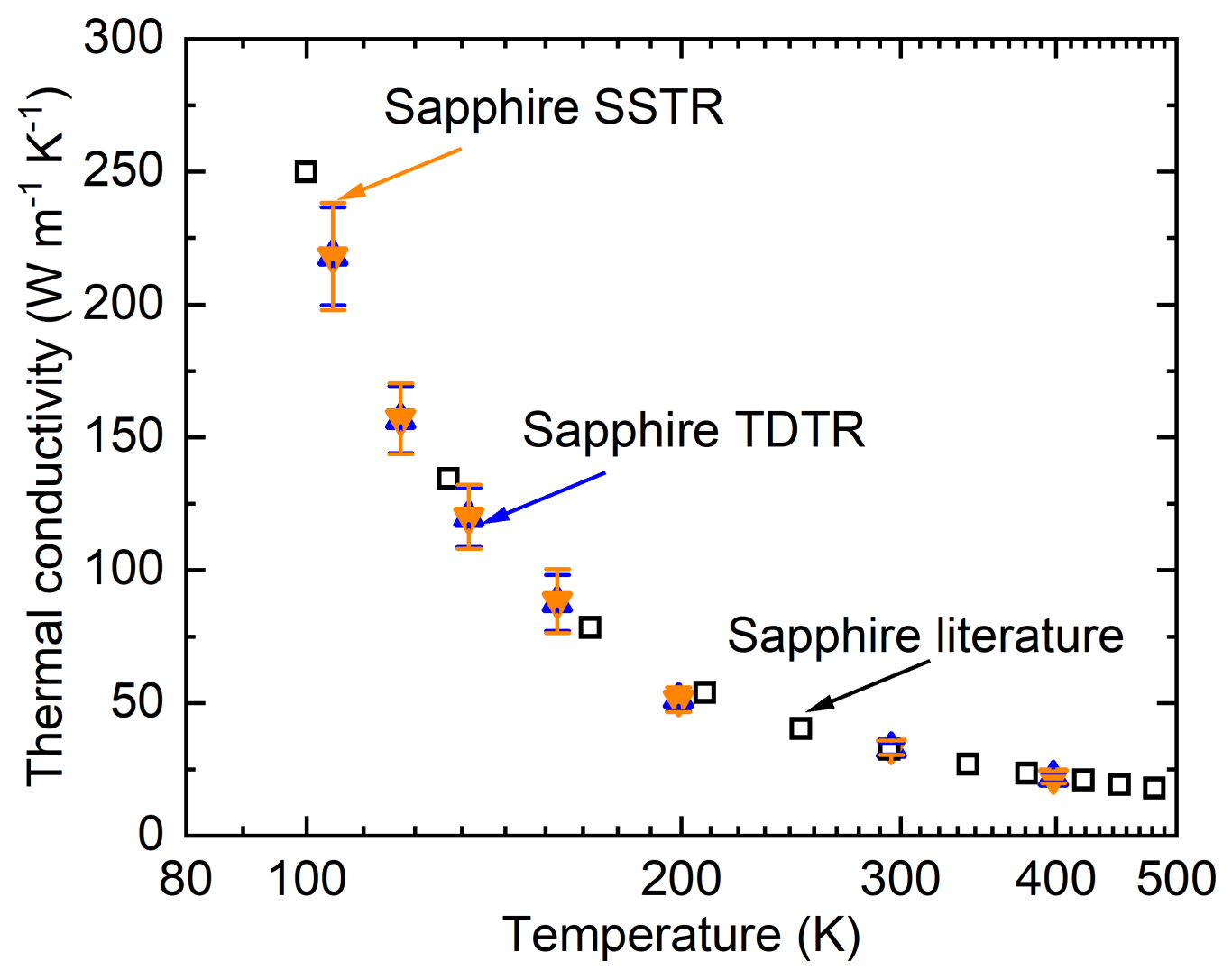

Figure S5: The TDTR-measured bulk sapphire thermal conductivities (blue filled upward triangles) used as references for determining the thermal conductivities of the $3.05 \mu \mathrm{m}$ AlN thin film by SSTR. From these TDTR-measured values, the $\gamma$ and corresponding uncertainty at each temperature is calculated. These $\gamma$ values are then used to determine the temperature-dependent in-plane thermal conductivities of the $3.05 \mu \mathrm{m}$ AlN film presented in Figure 4. The $\gamma$ values and corresponding uncertainty generate the exhibited SSTR-measured thermal conductivities of the sapphire reference (orange filled downward triangles). The TDTR-measured sapphire thermal conductivities are in agreement with the literature sapphire values ${ }^{20}$ (black open squares). 


\section{S8. SSTR measurements of thin film and anisotropic bulk control samples}

As stated in Section S5, for bulk materials, SSTR measures $\sqrt{(k \|)(k \perp)}$. However, for an insulating thin film on a conductive substrate, SSTR measurements represent $k_{\text {thin film }} \perp$. On the

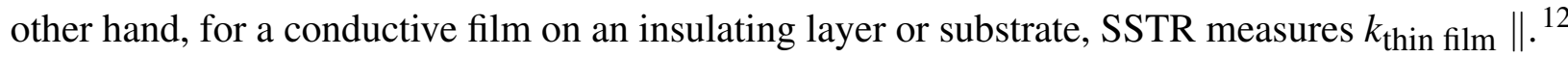
To experimentally show this, we choose three control samples: (i) bulk z-cut quartz, (ii) $102 \mathrm{~nm}$ amorphous silicon dioxide $\left(\mathrm{SiO}_{2}\right)$ thin film on a Si substrate, and (iii) $2.5 \mu \mathrm{m}$ Si thin film on $1 \mu \mathrm{m}$ $\mathrm{SiO}_{2}$ layer on top of a $\mathrm{Si}$ substrate. For measuring the thermal conductivity of these samples, $1 / \mathrm{e}^{2}$ pump and probe radii of $\sim 10 \mu \mathrm{m}$ are used.

Our first control sample is a bulk anisotropic material, $z$-cut quartz. The literature reported in-plane and cross-plane thermal conductivities of $z$-cut quartz are 6.5 and $11.6 \mathrm{~W} \mathrm{~m}^{-1} \mathrm{~K}^{-1}$, respectively. ${ }^{12,22,24}$ These reported values have a geometric mean of $\sqrt{6.5 \times 11.6}=8.68 \mathrm{~W} \mathrm{~m}^{-1}$ $\mathrm{K}^{-1}$. For comparison, the SSTR-measured thermal conductivity of $z$-cut quartz is $8.61 \pm 0.71 \mathrm{~W}$ $\mathrm{m}^{-1} \mathrm{~K}^{-1}$. This shows that for bulk materials, SSTR measures the geometric mean of in-plane and cross-plane thermal conductivities. Therefore, if either the in-plane or cross-plane thermal conductivity of the bulk material is known, then the other can be extracted from SSTR measurements. For example, if we adjust the in-plane thermal conductivity of $z$-cut quartz to be $6.5 \pm 0.65 \mathrm{~W}$ $\mathrm{m}^{-1} \mathrm{~K}^{-1}$ (10\% uncertainty), then the measured cross-plane thermal conductivity is $11.21 \pm 1.83$ $\mathrm{W} \mathrm{m} \mathrm{m}^{-1} \mathrm{~K}^{-1}$.

The second control sample is a $102 \mathrm{~nm} \mathrm{SiO}_{2}$ thin film on Si substrate. ${ }^{25}$ As the thermal conductivity of the thin film ${ }^{26,27}$ is significantly lower than that of substrate, ${ }^{28}$ SSTR measurements are overwhelmingly sensitive to the cross-plane thermal conductivity of the $\mathrm{SiO}_{2}$ film. ${ }^{12}$ Our SSTRmeasured thermal conductivity of the $\mathrm{SiO}_{2}$ thin film is $1.31 \pm 0.18 \mathrm{~W} \mathrm{~m}^{-1} \mathrm{~K}^{-1}$, in excellent agreement with literature. ${ }^{26,27}$ This value remains the same if the in-plane thermal conductivity of the $\mathrm{SiO}_{2}$ film is adjusted to 0.5 or $2 \mathrm{~W} \mathrm{~m}^{-1} \mathrm{~K}^{-1}$. This verifies that SSTR measurements are insensitive to the in-plane thermal conductivity of the $\mathrm{SiO}_{2}$ film, and the measured value represents $k_{\mathrm{SiO}_{2}} \perp$. 
The third control sample is a $2.5 \mu \mathrm{m}$ Si thin film on $1 \mu \mathrm{m} \mathrm{SiO}_{2}$ layer on top of Si substrate. For this sample, the in-plane thermal conductivity of the Si thin film dominates the sensitivity calculations when pump and probe radii of $10 \mu \mathrm{m}$ are used. ${ }^{29}$ The measured value of the Si film is $137 \pm 20 \mathrm{~W} \mathrm{~m}^{-1} \mathrm{~K}^{-1}$, in agreement with literature. ${ }^{28} \mathrm{We}$ achieve the same value if the cross-plane thermal conductivity of the Si film is adjusted to 50 or $200 \mathrm{~W} \mathrm{~m}^{-1} \mathrm{~K}^{-1}$, thus validating that SSTR is measuring the in-plane thermal conductivity of the film.

\section{S9. SSTR measurements of the single crystal AIN layer}

The thermal penetration depth of SSTR can be modulated by changing the pump radius. ${ }^{12}$ Therefore, using $1 / \mathrm{e}^{2}$ pump and probe radii of $\sim 3 \mu \mathrm{m},{ }^{30}$ we probe only the single crystal layer of the $6 \mu \mathrm{m}$ AlN thin film. The SSTR-measured thermal conductivity of this layer is $332 \pm 30 \mathrm{~W}$ $\mathrm{m}^{-1} \mathrm{~K}^{-1}$. This value is in agreement with the finding of Koh et al. ${ }^{3}$

Using large pump and probe radii, SSTR can measure the thermal conductivity of a buried layer when the thermal resistance of this layer is much higher compared to those of the top layer and substrate. ${ }^{29,31}$ Scott et al. ${ }^{31}$ showed this by measuring the thermal conductivity of an amorphous carbon layer in the following sample geometry: $\sim 7 \mu \mathrm{m}$ irradiated polycrystalline diamond/0.45 $\mu \mathrm{m}$ amorphous carbon layer/pristine polycrystalline diamond substrate. Similarly, the thermal conductivity of a buried $\mathrm{SiO}_{2}$ film in $\sim 2.5 \mu \mathrm{m} \mathrm{Si} / 1 \mu \mathrm{m} \mathrm{SiO}_{2}$ layer/Si substrate was measured by Hoque et al. ${ }^{29}$ using SSTR.

For the AlN thin films, the thickness of the nucleation layer is less than $22 \%$ of the total film thickness. Therefore, based on related literature, ${ }^{6,19,32-35}$ the thermal resistance of the nucleation layer can be expected to be relatively lower than those of the single crystal layer and sapphire substrate. Due to this, SSTR measurements cannot isolate the thermal conductivity of the buried nucleation layer. As a result, all SSTR measurements of the AIN films in this study corresponding to 10 and $20 \mu \mathrm{m}$ spot sizes represent in-plane thermal conductivities influenced by both single crystal and nucleation layers. 


\section{S10. Influence of interface on SSTR measurements}

TDTR measurements can be highly influenced by the interfacial-phonon scattering and inter-

face conditions. ${ }^{36}$ However, due to the measurement length scale, i.e., thermal penetration depth of the SSTR technique, it is much less influenced by the interface compared to TDTR. To show this, we have grown 12.05 and $26.3 \mathrm{~nm} \mathrm{SiO}_{2}$ on $\mathrm{Si}$ and measured the $\mathrm{Si}$ thermal conductivity by SSTR. Using a $\mathrm{SiO}_{2}$ thermal conductivity of $1.35 \mathrm{~W} \mathrm{~m}^{-1} \mathrm{~K}^{-1},{ }^{26,27}$ we analyze these two samples with a three layer model: $\mathrm{Al}$ transducer/SiO${ }_{2}$ thin film/Si. The measured $\mathrm{Si}$ values of $\mathrm{Al} / 12.05$ $\mathrm{nm} \mathrm{SiO}_{2} / \mathrm{Si}$ and $\mathrm{Al} / 26.3 \mathrm{~nm} \mathrm{SiO}_{2} / \mathrm{Si}$ are in agreement with the $\mathrm{Al} / \mathrm{Si}$ value as shown in Figure S6. For comparison, the $\mathrm{Si}$ thermal conductivities of $\mathrm{Al} / 11 \mathrm{~nm} \mathrm{SiO} 2 / \mathrm{Si}$ measured by TDTR from Wilson and Cahill ${ }^{36}$ are also presented. Due to the influence of interface, TDTR measurements of $\mathrm{Al} / 11 \mathrm{~nm} \mathrm{SiO} 2 / \mathrm{Si}$ exhibit a frequency dependence and decrease from $\sim 135$ to $122 \mathrm{~W} \mathrm{~m}^{-1} \mathrm{~K}^{-1}$ as modulation frequency increases from $\sim 1$ to $10 \mathrm{MHz}{ }^{36}$

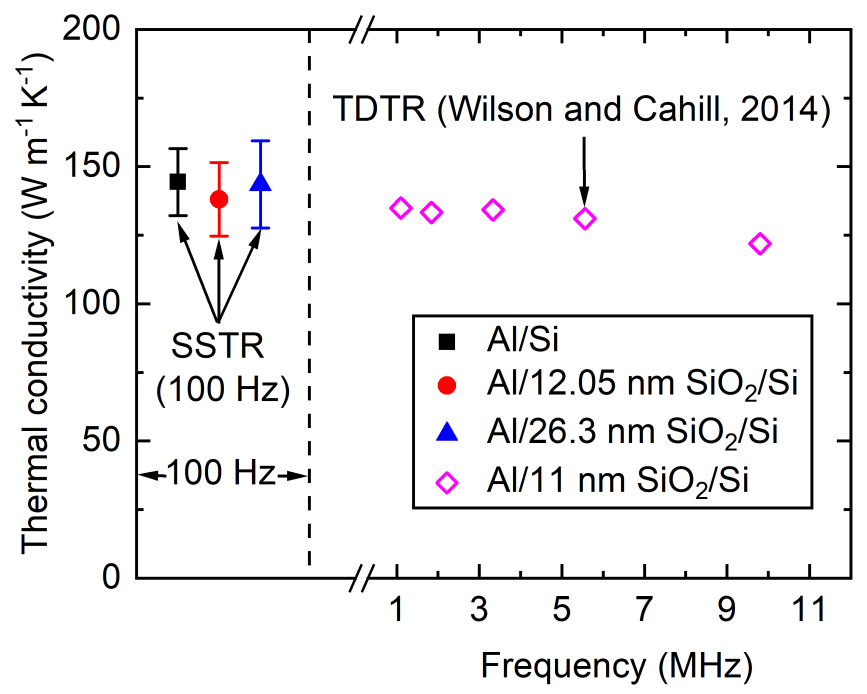

Figure S6: Influence of interface on Si thermal conductivity measurements by SSTR. The samples include $\mathrm{Al} / \mathrm{Si}, \mathrm{Al} / 12.05 \mathrm{~nm} \mathrm{SiO} 2 / \mathrm{Si}$, and $\mathrm{Al} / 26.3 \mathrm{~nm} \mathrm{SiO} 2 / \mathrm{Si}$. For comparison, the TDTRmeasured Si thermal conductivities of $\mathrm{Al} / 11 \mathrm{~nm} \mathrm{SiO}_{2} / \mathrm{Si}$ from Wilson and Cahill ${ }^{36}$ are also included. Filled symbols represent measurements taken in this study and open symbols represent literature values. 


\section{S11. TDTR measurements of the $3.05 \mu \mathrm{m}$ AIN thin film}

The AlN cross-plane thermal conductivities and Al/AlN thermal boundary conductances are determined by fitting a radially symmetric, multilayer heat diffusion model ${ }^{9,37-39}$ to the ratio of the in-phase and out-of-phase signal ( $\left.-V_{\text {in }} / V_{\text {out }}\right)$. Figure $\mathrm{S} 7 \mathrm{a}$ shows the best-fit thermal model to the TDTR data for the $3.05 \mu \mathrm{m}$ AlN thin film at $120 \mathrm{~K}$. At low temperatures, TDTR measurements have low sensitivity to the cross-plane thermal conductivity of the $3.05 \mu \mathrm{m} \mathrm{AlN} \mathrm{film.}{ }^{4}$ Moreover, the cross-plane thermal conductivity measurements are highly sensitive to the in-plane thermal conductivity at low temperatures. We show this in Figure S7b by plotting the sensitivity of TDTR measurements to the ratio $\left(-V_{\text {in }} / V_{\text {out }}\right)$ for the $3.05 \mu \mathrm{m}$ AlN film at $120 \mathrm{~K}$. A detailed description of the methodology used for this sensitivity calculation can be found in the literature. ${ }^{40-43}$ From Figure S7b, it is also evident that TDTR measurements have much higher sensitivity to the Al/AlN thermal boundary conductances.

We further recheck our AlN cross-plane thermal conductivities and Al/AlN thermal boundary conductances by fitting the thermal model to the in-phase signal $\left(V_{\text {in }}\right)$. The sensitivity analysis for this approach is shown in Figure S7c. As exhibited here, the in-phase signal has negligible sensitivity to the in-plane thermal conductivity. The AlN cross-plane thermal conductivities and Al/AlN thermal boundary conductances obtained from the fitting to the ratio, and in-phase signal are in good agreement.

In our previous work, ${ }^{3}$ it was demonstrated that modulation frequency and spot sizes do not have an appreciable influence on the cross-plane thermal conductivities of AlN thin films. Moreover, TDTR was used to measure the cross-plane thermal conductivity of a $17.5 \mu \mathrm{m}$ AlN film accurately down to $80 \mathrm{~K} .^{3}$ The same modulation frequency $(8.8 \mathrm{MHz})$ and spot sizes $\left(1 / \mathrm{e}^{2}\right.$ pump and probe radii of $\sim 8.5$ and $5 \mu \mathrm{m}$, respectively) are used to measure the cross-plane thermal conductivity of the $3.05 \mu \mathrm{m}$ AlN film in this study. Thus, it can be reasonably assumed that cross-plane thermal conductivities presented in Figure 5a are independent of any influence of TDTR modulation frequency and spot sizes. 

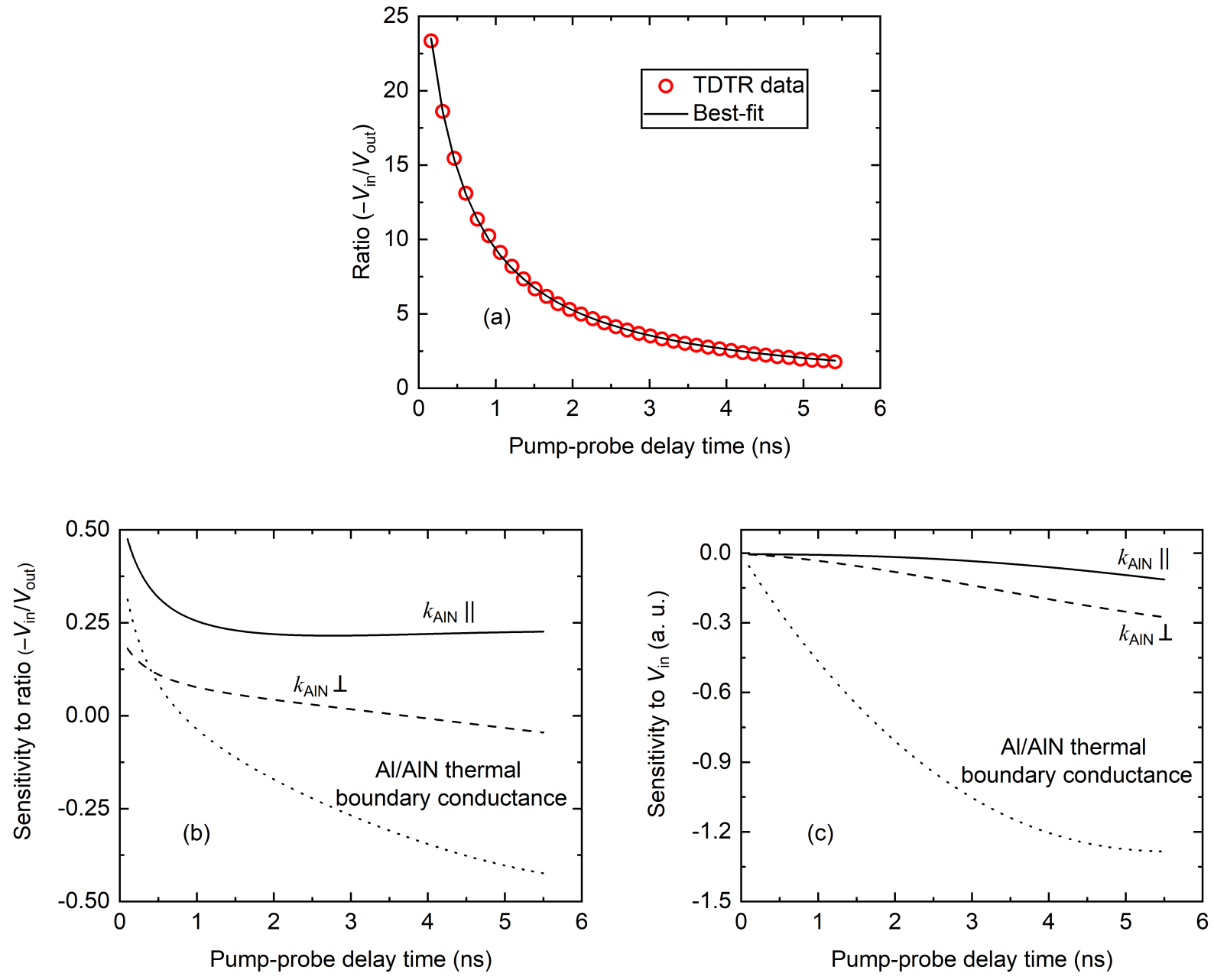

Figure S7: (a) Best-fit thermal model to the TDTR data for the $3.05 \mu \mathrm{m}$ AlN thin film at $120 \mathrm{~K}$. Sensitivity of TDTR measurements to the (b) ratio of in-phase and out-of-phase signal ( $-V_{\text {in }} / V_{\text {out }}$ ), and (c) in-phase signal $\left(V_{\text {in }}\right)$ for the $3.05 \mu \mathrm{m}$ AlN film at $120 \mathrm{~K}$. 


\section{S12. TDTR measurements of Si at low temperatures}

As shown in Figure 2, TDTR fails to measure the Si thermal conductivity at low temperatures. A $1 / \mathrm{e}^{2}$ pump radius of $\sim 8.5 \mu \mathrm{m}$ is used for our measurements. The Si thermal conductivities measured with this pump radius are lower than Wilson and Cahill's ${ }^{36} \mathrm{Si}$ measurements with 1/( ${ }^{2}$ pump radii of 5,10 , and $25 \mu \mathrm{m}$. Several factors can contribute to this difference observed between the Si thermal conductivities measured by us and ref. 36. The pump and probe radii used by ref. 36 were equal, however, our $1 / \mathrm{e}^{2}$ probe radius $(\sim 5 \mu \mathrm{m})$ is lower than the pump radius. Both pump and probe spot sizes are important length scales that can influence TDTR measurements of Si. ${ }^{36}$ Moreover, our Al/Si thermal boundary conductance $\left(175 \mathrm{MW} \mathrm{m}^{-2} \mathrm{~K}^{-1}\right)$ is lower than the value measured by ref. $36\left(250 \mathrm{MW} \mathrm{m}^{-2} \mathrm{~K}^{-1}\right)$ indicating different interface conditions. STEM characterization reveals a $3 \mathrm{~nm}$ native oxide layer in our Si sample, nearly the same as that of ref. 36 $(2 \mathrm{~nm})$. Therefore, the likely sources of the dissimilar interface conditions are the different surface treatments and $\mathrm{Al}$ deposition techniques. ${ }^{20,44-47}$ The Si sample of ref. 36 was heated to $\sim 600$ ${ }^{\circ} \mathrm{C}$, then cooled to room temperature in a high vacuum sputtering chamber prior to $\mathrm{Al}$ deposition. On the contrary, the Al deposition on our Si sample was done in an electron beam evaporator at room temperature and $1 \times 10^{-6}$ torr pressure without any heating. ${ }^{48}$ The resulting difference in the interface conditions can highly influence the Si thermal conductivity measurements by TDTR. ${ }^{36}$ We attribute these factors to be responsible for the discrepancy observed between us and ref. 36 .

We can rule out this discrepancy as an artifact of our TDTR setup by comparing our TDTR measured sapphire thermal conductivities with literature. As shown in Figure S5, our TDTR-measured sapphire thermal conductivities are in agreement with literature sapphire values. ${ }^{20}$ Moreover, the same TDTR setup was used to measure the temperature-dependent cross-plane thermal conductivity of a $17.5 \mu \mathrm{m}$ AlN film. ${ }^{3}$ Within uncertainty, the TDTR-measured values were in agreement with Slack et al.'s ${ }^{2}$ measurements of bulk, high-purity, single crystal AlN. These two measurements prove the capability of our TDTR setup to accurately measure the cross-plane thermal conductivity of the $3.05 \mu \mathrm{m}$ AlN film at low temperatures. 


\section{S13. Uncertainty analysis of TDTR and SSTR measurements}

For TDTR and SSTR measurements, uncertainty is calculated according to the following equa$\operatorname{tion}^{12}$

$$
\triangle=\sqrt{(\sigma)^{2}+\Sigma_{i} \triangle_{i}^{2}}
$$

Where $\triangle$ is the total uncertainty, $\sigma$ is the standard deviation among multiple measurements across different spots, and $\triangle_{i}$ is the uncertainty due to an individual parameter.

In Figure 2 and Figure S5, we present the thermal conductivities of bulk Si and sapphire determined by TDTR, respectively. The uncertainty of TDTR measurements incorporate the standard deviation among multiple measurements across different spots, the uncertainty associated with the transducer thickness, and the sapphire or Si heat capacity. The uncertainty associated with the TDTR-measured sapphire and Si thermal conductivities range from $\sim 8$ to $12 \%$. This range of uncertainty is typical in most TDTR measurements. ${ }^{1,12,36,49-51}$

The uncertainty of SSTR measurements presented in the main manuscript include the standard deviation, uncertainty associated with $\gamma, \mathrm{Al} / \mathrm{Si}$ or $\mathrm{Al} / \mathrm{AlN}$ thermal boundary conductance, and transducer thermal conductivity. At each temperature, $\gamma$ is determined from the TDTR-measured reference sapphire thermal conductivity. The uncertainty of the sapphire thermal conductivity propagates as the uncertainty of $\gamma$. Above $200 \mathrm{~K}$, the uncertainty of SSTR-measured Si and AlN thermal conductivities range from $\sim 8$ to $17 \%$. This range of uncertainty is in agreement with previous publications of SSTR measurements. ${ }^{3,52,53}$ Below $200 \mathrm{~K}$, however, due to increased SSTR measurement sensitivity to $\gamma$, and $\mathrm{Al} / \mathrm{Si}$ or $\mathrm{Al} / \mathrm{AlN}$ thermal boundary conductance, the uncertainty of $\mathrm{Si}$ and AlN thermal conductivities range from $\sim 18$ to $28 \%$.

The TDTR-measured cross-plane thermal conductivities of the $3.05 \mu \mathrm{m}$ AlN thin film presented in Figure 5a also have high uncertainty below $200 \mathrm{~K}$. The biggest source of this uncertainty is the in-plane thermal conductivity of the $3.05 \mu \mathrm{m}$ AlN film as shown in the sensitivity plot of Figure 
S7b. Due to the high uncertainty associated with the AlN in-plane thermal conductivities at low temperatures, TDTR cross-plane measurements also have higher uncertainty. Below $200 \mathrm{~K}$, the uncertainty of the $3.05 \mu \mathrm{m}$ AlN cross-plane thermal conductivities range from $\sim 40$ to $53 \%$. For low temperature in-plane and cross-plane thermal conductivity measurements of thin films, large uncertainty is not uncommon in literature..$^{4,19,54}$

The uncertainty of anisotropy ratio of the $3.05 \mu \mathrm{m}$ AlN film is calculated according to the following equation

$$
\frac{\triangle A}{\bar{A}}=\sqrt{\left(\frac{\triangle k \|}{\bar{k} \|}\right)^{2}+\left(\frac{\triangle k \perp}{\bar{k} \perp}\right)^{2}}
$$

Here, $\triangle \mathrm{A}, \bar{A}, \triangle k\|, \bar{k}\|, \triangle k \perp$ and $\bar{k} \perp$ represent the uncertainty of anisotropy ratio, mean anisotropy ratio, uncertainty of $k \|$, mean $k \|$, uncertainty of $k \perp$, and mean $k \perp$, respectively. 


\section{S14. Temperature-dependent thermal conductivity of thin film materials}

In Figure S8, we have plotted the temperature-dependent thermal conductivities of the $3.05 \mu \mathrm{m}$ AlN thin film, a $13 \mu \mathrm{m}$ diamond film, ${ }^{55}$ a $170 \mu \mathrm{m}$ isotopically enriched diamond film, ${ }^{56}$ a $15 \pm$ $2 \mu \mathrm{m} 78 \%{ }^{10} \mathrm{~B}$ (near-natural) $\mathrm{hBN}$ film,,${ }^{54}$ and a $19 \pm 2 \mu \mathrm{m}$ pyrolytic graphite film. ${ }^{57}$ With the exception of the $3.05 \mu \mathrm{m}$ AlN film, the rest of the thin film materials exhibit a thermal conductivity reduction with temperature decrease due to defect and boundary scattering. However, since the inplane thermal transport mechanisms of our AlN thin films are primarily driven by phonon-phonon scattering, the in-plane thermal conductivity of the $3.05 \mu \mathrm{m}$ film increases with a reduction in temperature.

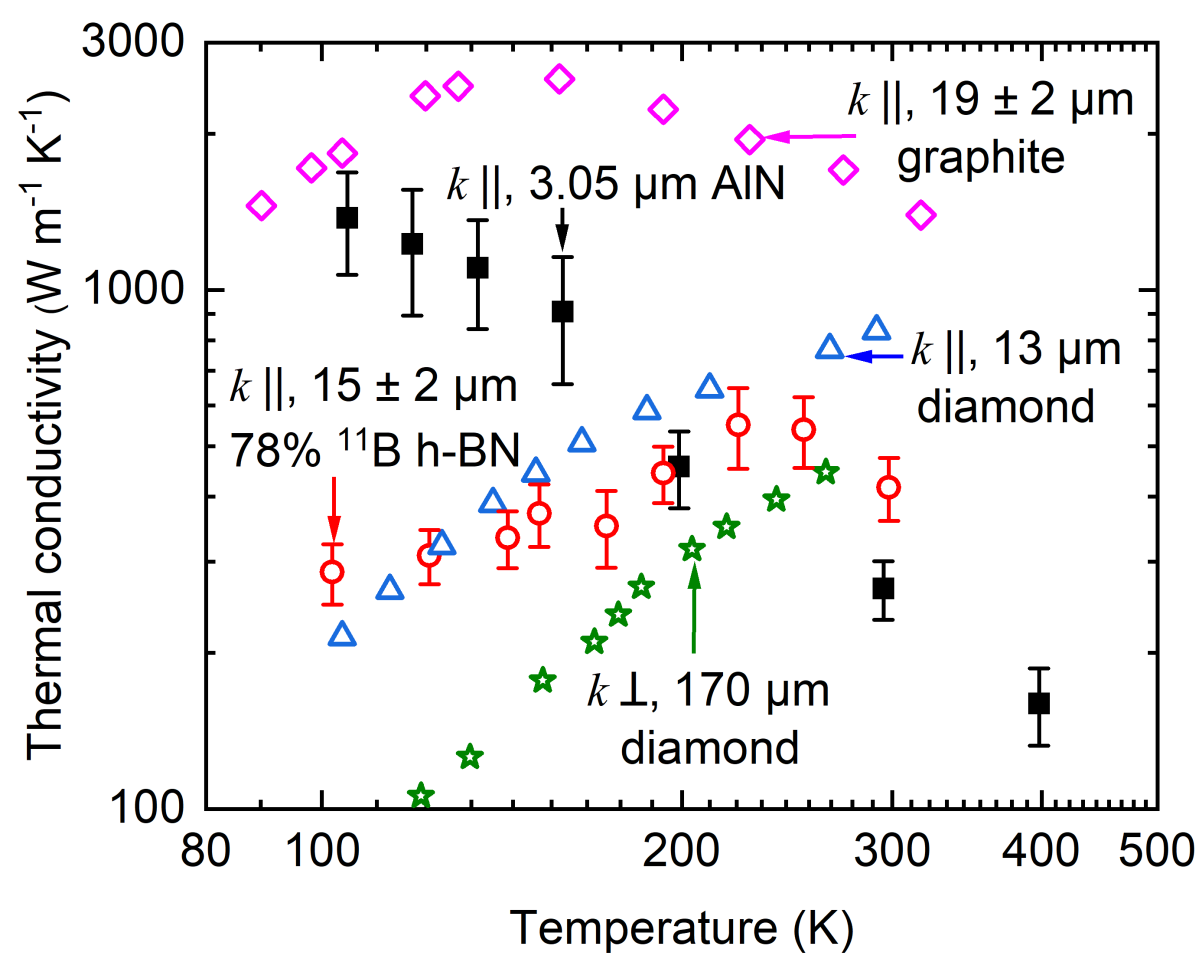

Figure S8: Temperature-dependent in-plane and cross-plane thermal conductivities of several high thermal conductivity materials: $3.05 \mu \mathrm{m}$ AlN film, $13 \mu \mathrm{m}$ diamond film, ${ }^{55} 170 \mu \mathrm{m}$ isotopically enriched diamond film, ${ }^{56} 15 \pm 2 \mu \mathrm{m} 78 \%{ }^{10} \mathrm{~B}$ (near-natural) $\mathrm{hBN}$ film, ${ }^{54}$ and $19 \pm 2 \mu \mathrm{m}$ pyrolytic graphite film. ${ }^{57}$ Filled symbols represent measurements taken in this study and open symbols represent literature values. 


\section{S15. Boltzmann transport equation (BTE) method used to calculate the AlN mean-free-path spectra}

In Figure S9, the AlN cross-plane thermal conductivity accumulations as a function of phonon mean-free-path spectra at room temperature, 105 and $120 \mathrm{~K}$ are presented. To obtain this, an iterative scheme is applied to solve the linearized phonon Boltzmann transport equation with the help of first-principles force constants. We first relax the AIN atomic structure to its optimized positions using Quantum Espresso. ${ }^{58}$ Then second-order force constants which provide harmonic phonon properties, are calculated using Density Functional Perturbation Theory (DFPT), in an discretized $4 \times 4 \times 4$ q space grid. Beyond harmonic approximation, three-phonon scattering process is considered with the help of the third-order force constants calculated in a finite different scheme on a supercell. Phonon-isotope scattering is further considered. With the help of these first-principles force constants, the linearized phonon BTE is solved iteratively using ShengBTE 59 in a $12 \times 12 \times 12$ grid.

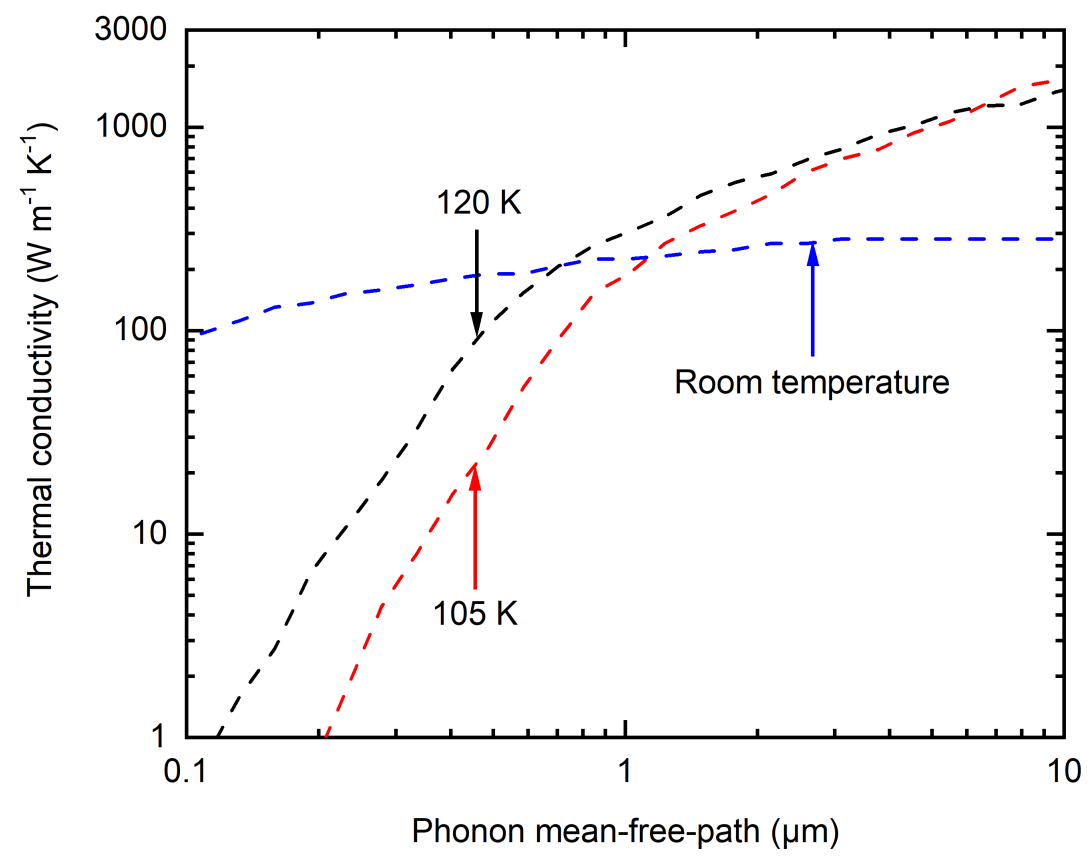

Figure S9: The AlN cross-plane thermal conductivity accumulations as a function of phonon mean-free-path spectra at room temperature, 105 and $120 \mathrm{~K}$. 


\section{S16. A simplified thermal resistance model for the $3.05 \mu \mathrm{m}$ AIN film}

The anisotropy in the thermal conductivity of the $3.05 \mu \mathrm{m}$ AlN film can be described by a macroscopic thermal resistance model. This is shown here by calculating the anisotropy ratios at room temperature and $120 \mathrm{~K}$. This resistance model considers the AlN thin films to be consisted of two different thermal conductivity layers separated by an interface. Assuming the interface conductance to be very high, the absolute thermal resistance ${ }^{60}(R)$ of each layer can be expressed as the following

$$
R=\frac{L}{k A}
$$

Here, $L$ represents the length scale along the path of heat flow, whereas $A$ denotes crosssectional area perpendicular to the path of heat flow. Figure S10 shows the approximated heat flow directions during the cross-plane and in-plane thermal conductivity measurements of the AlN films. As exhibited here, $L$ and $A$ are not the same for cross-plane and in-plane measurements. For simplicity, we consider $A$ of both layers to be the same for cross-plane measurements, whereas $L$ remains constant for in-plane measurements.

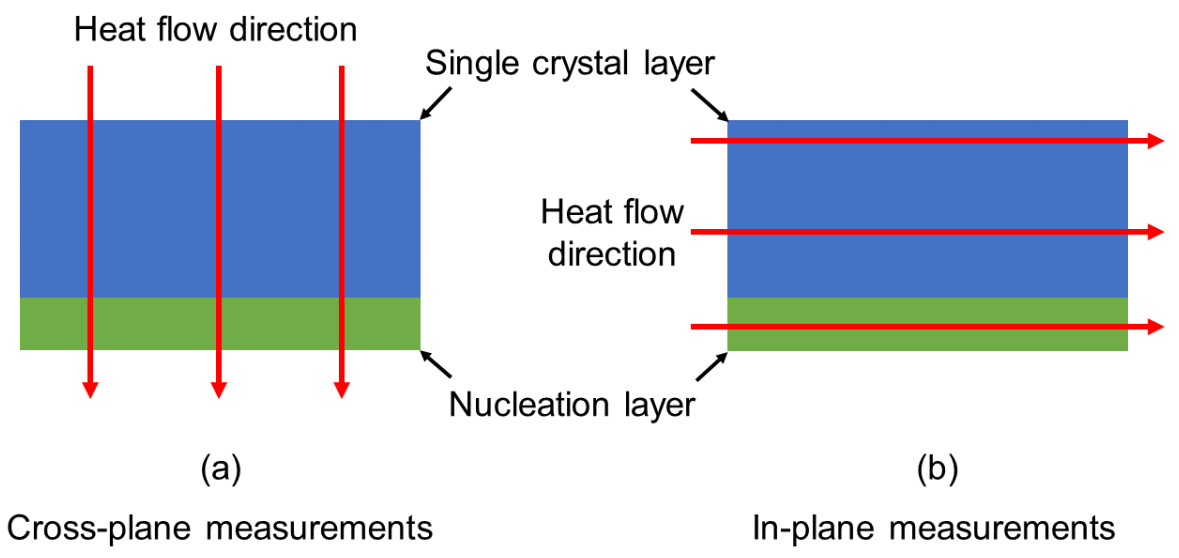

Figure S10: Approximated heat flow directions during (a) cross-plane and (b) in-plane thermal conductivity measurements of the AlN films.

TDTR and SSTR probe through both layers of the $3.05 \mu \mathrm{m}$ AIN film. Thus, the TDTR and 
SSTR measurements presented in Figure 5a can be considered as effective thermal conductivities. Using a series resistor model, ${ }^{48}$ the following expression can be obtained for the effective crossplane thermal conductivity

$$
\left(\frac{t}{k \perp}\right)_{\text {effective }}=\left(\frac{t}{k \perp}\right)_{\text {single crystal layer }}+\left(\frac{t}{k \perp}\right)_{\text {nucleation layer }}
$$

Here, $t$ denotes thickness. Similarly, using a parallel resistor model, ${ }^{61}$ the effective in-plane thermal conductivity can be expressed as follows

$$
\{t \times(k \|)\}_{\text {effective }}=\{t \times(k \|)\}_{\text {single crystal layer }}+\{t \times(k \|)\}_{\text {nucleation layer }}
$$

Though the thermal conductivity of the single crystal layer is known at room temperature (Section S9), it is an unknown parameter at $120 \mathrm{~K}$. To estimate this thermal conductivity, we use the AlN mean-free-path spectra presented in Figure S9. In the single crystal layer, the average distance traveled by the cross-plane phonons before scattering at the interface is nearly the same as the layer thickness, but for the in-plane phonons, it is many times that thickness. 4,16,17,62 Thus, we assume that at $120 \mathrm{~K}$, the cross-plane thermal conductivity corresponds to a mean-free-path equivalent to the layer thickness $(\sim 2.45 \mu \mathrm{m})$, whereas the in-plane thermal conductivity corresponds to the highest mean-free-path $(\sim 10 \mu \mathrm{m})$ shown in Figure S9. We note that the thermal conductivities obtained under this assumption are highly approximated values used for the simplification of calculations.

As discussed in the main manuscript, the thermal conductivity of the nucleation layer can be expected to remain near isotropic across all temperatures of interest in this study. As this layer contains high concentrations of point and line defects, we can only estimate an upper limit of the nucleation layer thermal conductivity. We assume that the phonons are being scattered after traveling uninterrupted throughout the entire thickness of this layer $(\sim 0.6 \mu \mathrm{m})$. Therefore, both at room temperature and $120 \mathrm{~K}$, the cross-plane and in-plane thermal conductivities of the nucleation 
layer correspond to a mean-free-path of $0.6 \mu \mathrm{m}$.

Using equations (S10 and S11) under the above-mentioned assumptions, at room temperature, the effective cross-plane and in-plane thermal conductivities are calculated to be $\sim 282$ and $295 \mathrm{~W}$ $\mathrm{m}^{-1} \mathrm{~K}^{-1}$, respectively. These values provide an anisotropy ratio of 1.05 . Similarly, at $120 \mathrm{~K}$, the calculated effective cross-plane and in-plane thermal conductivities are $\sim 397$ and $1253 \mathrm{~W} \mathrm{~m}^{-1}$ $\mathrm{K}^{-1}$, respectively with an anisotropy ratio of 3.16 .

The calculated thermal conductivities and anisotropy ratios are in agreement with the experimentally measured values presented in Figures 5a and 5c. This shows that a macroscopic thermal resistance model can describe the anisotropy in the thermal conductivity of the $3.05 \mu \mathrm{m}$ AlN thin film. However, it is to be noted that the model we use is highly simplified due to the assumptions and approximations made here. To properly account for the anisotropy of the $3.05 \mu \mathrm{m}$ film, a rigorous model with more known parameters is needed which is beyond the scope of this work. 
Table S1: Parameters used in the measurements of room-temperature in-plane thermal conductivities of the AlN thin films. During the sensitivity calculations of Figure S2, the average values presented here are used.

\begin{tabular}{|c|c|c|c|}
\hline Parameters & $3.05 \mu \mathrm{m}$ film & $3.75 \mu \mathrm{m}$ film & $6 \mu \mathrm{m}$ film \\
\hline $\begin{array}{c}\text { Al transducer thickness } \\
(\mathrm{nm})\end{array}$ & $77 \pm 3$ & $77 \pm 3$ & $83 \pm 3$ \\
\hline $\begin{array}{c}\text { a } \\
\text { Al/AlN thermal boundary conductance } \\
\left(\mathrm{MW} \mathrm{m}^{-2} \mathrm{~K}^{-1}\right)\end{array}$ & $120 \pm 18$ & $95 \pm 14$ & $145 \pm 20$ \\
\hline $\begin{array}{c}\text { AlN/sapphire thermal boundary conductance } \\
\left(\mathrm{MW} \mathrm{m}^{-2} \mathrm{~K}^{-1}\right)\end{array}$ & 200 & 200 & 200 \\
\hline $\begin{array}{c}\text { Al transducer thermal conductivity } \\
(\mathrm{W} \mathrm{m}\end{array}$ & $130 \pm 20$ & $130 \pm 20$ & $130 \pm 20$ \\
\hline
\end{tabular}

${ }^{\text {a }}$ Determined by picosecond acoustics. ${ }^{63}$

${ }^{b}$ Measured by TDTR.

${ }^{c}$ SSTR measurements are not sensitive to the AlN/sapphire thermal boundary conductances. Adjusting this value to 50 or $350 \mathrm{MW} \mathrm{m}^{-2} \mathrm{~K}^{-1}$ results in negligible changes to the sensitivity calculations and measured in-plane thermal conductivities.

${ }^{\mathrm{d}}$ Determined by 4-point probe. 
Table S2: Bulk in-plane thermal conductivities of the thin film materials presented in Figure $3 b$. For hBN, the predicted in-plane thermal conductivity of defect-free, bulk material is adopted as the bulk value. For Si, diamond, AlN, and GaN, bulk in-plane and cross-plane thermal conductivities are considered to be the same..$^{5,22,64,65}$

\begin{tabular}{|c|c|c|}
\hline Materials & $\begin{array}{l}\text { Bulk in-plane thermal conductivity } \\
\qquad\left(\mathrm{Wm}^{-1} \mathrm{~K}^{-1}\right)\end{array}$ & Reference \\
\hline $\mathrm{Si}$ & 151 & Touloukian et al. ${ }^{66}$ \\
\hline Diamond & 2200 & Zheng et al. ${ }^{67}$ \\
\hline $\begin{array}{l}\text { Highly oriented pyrolytic graphite } \\
\qquad(\mathrm{HOPG})\end{array}$ & 2054 & Li et al. ${ }^{22}$ \\
\hline AlN & 323 & Koh et al. ${ }^{3}$ \\
\hline $\mathrm{GaN}$ & 253 & Shibata et al. ${ }^{68}$ \\
\hline $99 \%{ }^{10} \mathrm{~B} \mathrm{hBN}$ & 611 & Yuan et al. ${ }^{54}$ \\
\hline $99 \%{ }^{11} \mathrm{~B} \mathrm{hBN}$ & 611 & Yuan et al. ${ }^{54}$ \\
\hline $78 \%{ }^{11} \mathrm{~B} \mathrm{hBN}$ (natural) & 555 & Yuan et al. ${ }^{54}$ \\
\hline $48 \%{ }^{11} \mathrm{~B} \mathrm{hBN}$ & 515 & Yuan et al. ${ }^{54}$ \\
\hline
\end{tabular}




\section{References}

1. Cheng, Z.; Koh, Y. R.; Mamun, A.; Shi, J.; Bai, T.; Huynh, K.; Yates, L.; Liu, Z.; Li, R.; Lee, E.; Liao, M. E.; Wang, Y.; Yu, H. M.; Kushimoto, M.; Luo, T.; Goorsky, M. S.; Hopkins, P. E.; Amano, H.; Khan, A.; Graham, S. Experimental Observation of High Intrinsic Thermal Conductivity of AlN. Phys. Rev. Mater. 2020, 4, 044602.

2. Slack, G. A.; Tanzilli, R. A.; Pohl, R.; Vandersande, J. The Intrinsic Thermal Conductivity of AlN. J. Phys. Chem. Solids 1987, 48, 641-647.

3. Koh, Y. R.; Cheng, Z.; Mamun, A.; Bin Hoque, M. S.; Liu, Z.; Bai, T.; Hussain, K.; Liao, M. E.; Li, R.; Gaskins, J. T.; Giri, A.; Tomko, J.; Braun, J. L.; Gaevski, M.; Lee, E.; Yates, L.; Goorsky, M. S.; Luo, T.; Khan, A.; Graham, S. et al. Bulk-Like Intrinsic Phonon Thermal Conductivity of Micrometer Thick AlN Films. ACS Appl. Mater. Interfaces 2020, 12, 29443-29450.

4. Sun, B.; Haunschild, G.; Polanco, C.; Ju, J. Z.-J.; Lindsay, L.; Koblmüller, G.; Koh, Y. K. Dislocation-Induced Thermal Transport Anisotropy in Single-Crystal Group-III Nitride Films. Nat. Mater. 2019, 18, 136-140.

5. Lindsay, L.; Broido, D.; Reinecke, T. Ab Initio Thermal Transport in Compound Semiconductors. Phys. Rev. B 2013, 87, 165201.

6. Xu, R. L.; Muñoz Rojo, M.; Islam, S.; Sood, A.; Vareskic, B.; Katre, A.; Mingo, N.; Goodson, K. E.; Xing, H. G.; Jena, D.; Pop, E. Thermal Conductivity of Crystalline AlN and the Influence of Atomic-Scale Defects. J. Appl. Phys. 2019, 126, 185105.

7. Rounds, R.; Sarkar, B.; Sochacki, T.; Bockowski, M.; Imanishi, M.; Mori, Y.; Kirste, R.; Collazo, R.; Sitar, Z. Thermal Conductivity of GaN Single Crystals: Influence of Impurities Incorporated in Different Growth Processes. J. Appl. Phys. 2018, 124, 105106. 
8. Rounds, R.; Sarkar, B.; Alden, D.; Guo, Q.; Klump, A.; Hartmann, C.; Nagashima, T.; Kirste, R.; Franke, A.; Bickermann, M.; Kumagai, Y.; Sitar, Z.; Collazo, R. The Influence of Point Defects on the Thermal Conductivity of AlN Crystals. J. Appl. Phys. 2018, 123, 185107.

9. Cahill, D. G. Analysis of Heat Flow in Layered Structures for Time-Domain Thermoreflectance. Rev. Sci. Instrum. 2004, 75, 5119-5122.

10. Braun, J. L.; Hopkins, P. E. Upper Limit to the Thermal Penetration Depth during Modulated Heating of Multilayer Thin Films with Pulsed and Continuous Wave Lasers: A Numerical Study. J. Appl. Phys. 2017, 121, 175107.

11. Braun, J. L.; Szwejkowski, C. J.; Giri, A.; Hopkins, P. E. On the Steady-State Temperature Rise during Laser Heating of Multilayer Thin Films in Optical Pump-Probe Techniques. $J$. Heat Transfer 2018, 140, 052801.

12. Braun, J. L.; Olson, D. H.; Gaskins, J. T.; Hopkins, P. E. A Steady-State Thermoreflectance Method to Measure Thermal Conductivity. Rev. Sci. Instrum. 2019, 90, 024905.

13. Yang, J.; Maragliano, C.; Schmidt, A. J. Thermal Property Microscopy with Frequency Domain Thermoreflectance. Rev. Sci. Instrum. 2013, 84, 104904.

14. Slack, G.; McNelly, T. AlN Single Crystals. J. Cryst. Growth 1977, 42, 560-563.

15. Rounds, R.; Sarkar, B.; Klump, A.; Hartmann, C.; Nagashima, T.; Kirste, R.; Franke, A.; Bickermann, M.; Kumagai, Y.; Sitar, Z.; Collazo, R. Thermal Conductivity of Single-Crystalline AlN. Appl. Phys. Express 2018, 11, 071001.

16. Gomes, C. J.; Madrid, M.; Goicochea, J. V.; Amon, C. H. In-Plane and Out-of-Plane Thermal Conductivity of Silicon Thin Films Predicted by Molecular Dynamics. J. Heat Transfer 2006, $128,1114-1121$.

17. Jeong, C.; Datta, S.; Lundstrom, M. Thermal Conductivity of Bulk and Thin-Film Silicon: A Landauer Approach. J. Appl. Phys. 2012, 111, 093708. 
18. Kwon, S.; Zheng, J.; Wingert, M. C.; Cui, S.; Chen, R. Unusually High and Anisotropic Thermal Conductivity in Amorphous Silicon Nanostructures. ACS nano 2017, 11, 2470-2476.

19. Li, H.; Hanus, R.; Polanco, C. A.; Zeidler, A.; Koblmüller, G.; Koh, Y. K.; Lindsay, L. GaN Thermal Transport Limited by the Interplay of Dislocations and Size Effects. Phys. Rev. B 2020, 102, 014313.

20. Cheng, Z.; Koh, Y. R.; Ahmad, H.; Hu, R.; Shi, J.; Liao, M. E.; Wang, Y.; Bai, T.; Li, R.; Lee, E.; Clinton, E. A.; Matthews, C. M.; Engel, Z.; Yates, L.; Luo, T.; Goorsky, M. S.; Doolittle, W. A.; Tian, Z.; Hopkins, P. E.; Graham, S. Thermal Conductance across HarmonicMatched Epitaxial Al-Sapphire Heterointerfaces. Commun. Phys. 2020, 3, 1-8.

21. Dong, Y.; Cao, B.-Y.; Guo, Z.-Y. Ballistic-Diffusive Phonon Transport and Size Induced Anisotropy of Thermal Conductivity of Silicon Nanofilms. Phys. E 2015, 66, 1-6.

22. Li, M.; Kang, J. S.; Hu, Y. Anisotropic Thermal Conductivity Measurement Using a New Asymmetric-Beam Time-Domain Thermoreflectance (AB-TDTR) Method. Rev. Sci. Instrum. 2018, 89, 084901.

23. Monchamp, R. R. Preparation and Properties of Crystalline Laser Oxide Materials. J. Solid State Chem. 1975, 12, 201-206.

24. Feser, J. P.; Liu, J.; Cahill, D. G. Pump-Probe Measurements of the Thermal Conductivity Tensor for Materials Lacking In-Plane Symmetry. Rev. Sci. Instrum. 2014, 85, 104903.

25. Braun, J. L.; Baker, C. H.; Giri, A.; Elahi, M.; Artyushkova, K.; Beechem, T. E.; Norris, P. M.; Leseman, Z. C.; Gaskins, J. T.; Hopkins, P. E. Size Effects on the Thermal Conductivity of Amorphous Silicon Thin Films. Phys. Rev. B 2016, 93, 140201.

26. Cahill, D. G. Thermal Conductivity Measurement from 30 to $750 \mathrm{~K}$ : The $3 \omega$ Method. Rev. Sci. Instrum. 1990, 61, 802-808. 
27. Giri, A.; Chen, A. Z.; Mattoni, A.; Aryana, K.; Zhang, D.; Hu, X.; Lee, S.-H.; Choi, J. J.; Hopkins, P. E. Ultralow Thermal Conductivity of Two-Dimensional Metal Halide Perovskites. Nano Lett. 2020, 20, 3331-3337.

28. Marconnet, A. M.; Asheghi, M.; Goodson, K. E. From the Casimir Limit to Phononic Crystals: 20 Years of Phonon Transport Studies Using Silicon-on-Insulator Technology. J. Heat Transfer 2013, $135,061601$.

29. Hoque, M. S. B.; Koh, Y. R.; Aryana, K.; Hoglund, E.; Braun, J. L.; Olson, D. H.; Gaskins, J. T.; Ahmad, H.; Elahi, M. M. M.; Hite, J. K.; Leseman, Z. C.; Doolittle, W. A.; Hopkins, P. E. Thermal Conductivity Measurements of Sub-Surface Buried Substrates by SteadyState Thermoreflectance. arXiv preprint arXiv:2102.12954 2021, 1-17.

30. Giri, A.; Chou, S. S.; Drury, D. E.; Tomko, K. Q.; Olson, D.; Gaskins, J. T.; Kaehr, B.; Hopkins, P. E. Molecular Tail Chemistry Controls Thermal Transport in Fullerene Films. Phys. Rev. Mater. 2020, 4, 065404.

31. Scott, E. A.; Braun, J. L.; Hattar, K.; Sugar, J. D.; Gaskins, J. T.; Goorsky, M.; King, S. W.; Hopkins, P. E. Probing Thermal Conductivity of Subsurface, Amorphous Layers in Irradiated Diamond. J. Appl. Phys. 2021, 129, 055307.

32. Qiao, L.; Zhou, H.; Xue, H.; Wang, S. Effect of $\mathrm{Y}_{2} \mathrm{O}_{3}$ on Low Temperature Sintering and Thermal Conductivity of AlN Ceramics. J. Eur. Ceram. Soc. 2003, 23, 61-67.

33. Kume, S.; Yasuoka, M.; Lee, S.-K.; Kan, A.; Ogawa, H.; Watari, K. Dielectric and Thermal Properties of AlN Ceramics. J. Eur. Ceram. Soc. 2007, 27, 2967-2971.

34. AlShaikhi, A.; Srivastava, G. Thermal Conductivity of Single Crystal and Ceramic AlN. $J$. Appl. Phys. 2008, 103, 083554.

35. Kusunose, T.; Sekino, T. Improvement in Fracture Strength in Electrically Conductive AIN Ceramics with High Thermal Conductivity. Ceram. Int. 2016, 42, 13183-13189. 
36. Wilson, R.; Cahill, D. G. Anisotropic Failure of Fourier Theory in Time-Domain Thermoreflectance Experiments. Nat. Commun. 2014, 5, 5075.

37. Hopkins, P. E.; Serrano, J. R.; Phinney, L. M.; Kearney, S. P.; Grasser, T. W.; Harris, C. T. Criteria for Cross-Plane Dominated Thermal Transport in Multilayer Thin Film Systems during Modulated Laser Heating. J. Heat Transfer 2010, 132, 081302.

38. Schmidt, A. J.; Chen, X.; Chen, G. Pulse Accumulation, Radial Heat Conduction, and Anisotropic Thermal Conductivity in Pump-Probe Transient Thermoreflectance. Rev. Sci. Instrum. 2008, 79, 114902.

39. Cahill, D. G.; Goodson, K.; Majumdar, A. Thermometry and Thermal Transport in Micro/Nanoscale Solid-State Devices and Structures. J. Heat Transfer 2002, 124, 223-241.

40. Koh, Y. K.; Singer, S. L.; Kim, W.; Zide, J. M.; Lu, H.; Cahill, D. G.; Majumdar, A.; Gossard, A. C. Comparison of the $3 \omega$ Method and Time-Domain Thermoreflectance for Measurements of the Cross-Plane Thermal Conductivity of Epitaxial Semiconductors. J. Appl. Phys. 2009, 105, 054303.

41. Jiang, P.; Huang, B.; Koh, Y. K. Accurate Measurements of Cross-Plane Thermal Conductivity of Thin Films by Dual-Frequency Time-Domain Thermoreflectance (TDTR). Rev. Sci. Instrum. 2016, 87, 075101.

42. Giri, A.; Niemelä, J.-P.; Tynell, T.; Gaskins, J. T.; Donovan, B. F.; Karppinen, M.; Hopkins, P. E. Heat-Transport Mechanisms in Molecular Building Blocks of Inorganic/Organic Hybrid Superlattices. Phys. Rev. B 2016, 93, 115310.

43. Giri, A.; Niemelä, J.-P.; Szwejkowski, C. J.; Karppinen, M.; Hopkins, P. E. Reduction in Thermal Conductivity and Tunable Heat Capacity of Inorganic/Organic Hybrid Superlattices. Phys. Rev. B 2016, 93, 024201. 
44. Hopkins, P. E.; Norris, P. M.; Stevens, R. J.; Beechem, T. E.; Graham, S. Influence of Interfacial Mixing on Thermal Boundary Conductance across a Chromium/Silicon Interface. J. Heat Transfer 2008, 130, 062402.

45. Collins, K. C.; Chen, S.; Chen, G. Effects of Surface Chemistry on Thermal Conductance at Aluminum-Diamond Interfaces. Appl. Phys. Lett. 2010, 97, 083102.

46. Monachon, C.; Weber, L. Influence of Diamond Surface Termination on Thermal Boundary Conductance between Al and Diamond. J. Appl. Phys. 2013, 113, 183504.

47. Monachon, C.; Weber, L.; Dames, C. Thermal Boundary Conductance: A Materials Science Perspective. Annu. Rev. Mater. Sci. 2016, 46, 433-463.

48. DeCoster, M. E.; Chen, X.; Zhang, K.; Rost, C. M.; Hoglund, E. R.; Howe, J. M.; Beechem, T. E.; Baumgart, H.; Hopkins, P. E. Thermal Conductivity and Phonon Scattering Processes of ALD Grown PbTe-PbSe Thermoelectric Thin Films. Adv. Funct. Mater. 2019, 29, 1904073.

49. Wilson, R.; Cahill, D. G. Limits to Fourier Theory in High Thermal Conductivity Single Crystals. Appl. Phys. Lett. 2015, 107, 203112.

50. Braun, J. L.; Rost, C. M.; Lim, M.; Giri, A.; Olson, D. H.; Kotsonis, G. N.; Stan, G.; Brenner, D. W.; Maria, J.-P.; Hopkins, P. E. Charge-Induced Disorder Controls the Thermal Conductivity of Entropy-Stabilized Oxides. Adv. Mater. 2018, 30, 1805004.

51. Gaskins, J. T.; Kotsonis, G.; Giri, A.; Ju, S.; Rohskopf, A.; Wang, Y.; Bai, T.; Sachet, E.; Shelton, C. T.; Liu, Z.; Cheng, Z.; Foley, B. M.; Graham, S.; Luo, T.; Henry, A.; Goorsky, M. S.; Shiomi, J.; Maria, J.-P.; Hopkins, P. E. Thermal Boundary Conductance across Heteroepitaxial ZnO/GaN Interfaces: Assessment of the Phonon Gas Model. Nano Lett. 2018, 18, 7469-7477.

52. Qin, M.; Gild, J.; Hu, C.; Wang, H.; Hoque, S. B.; Braun, J. L.; Harrington, T. J.; Hop- 
kins, P. E.; Vecchio, K. S.; Luo, J. Dual-Phase High-Entropy Ultrahigh Temperature Ceramics. J. Eur. Ceram. Soc. 2020, 40, 5037 - 5050.

53. Jang, E.; Banerjee, P.; Huang, J.; Holley, R.; Gaskins, J. T.; Hoque, M. S. B.; Hopkins, P. E.; Madan, D. Thermoelectric Performance Enhancement of Naturally Occurring Bi and Chitosan Composite Films Using Energy Efficient Method. Electronics 2020, 9, 532.

54. Yuan, C.; Li, J.; Lindsay, L.; Cherns, D.; Pomeroy, J. W.; Liu, S.; Edgar, J. H.; Kuball, M. Modulating the Thermal Conductivity in Hexagonal Boron Nitride via Controlled Boron Isotope Concentration. Commun. Phys. 2019, 2, 1-8.

55. Morelli, D.; Beetz, C.; Perry, T. Thermal Conductivity of Synthetic Diamond Films. J. Appl. Phys. 1988, 64, 3063-3066.

56. Anthony, T.; Fleischer, J.; Olson, J.; Cahill, D. G. The Thermal Conductivity of Isotopically Enriched Polycrystalline Diamond Films. J. Appl. Phys. 1991, 69, 8122-8125.

57. Nakamura, S.; Miyafuji, D.; Fujii, T.; Matsui, T.; Fukuyama, H. Low Temperature Transport Properties of Pyrolytic Graphite Sheet. Cryogenics 2017, 86, 118-122.

58. Giannozzi, P.; Baroni, S.; Bonini, N.; Calandra, M.; Car, R.; Cavazzoni, C.; Ceresoli, D.; Chiarotti, G. L.; Cococcioni, M.; Dabo, I.; Dal Corso, A.; de Gironcoli, S.; Fabris, S.; Fratesi, G.; Gebauer, R.; Gerstmann, U.; Gougoussis, C.; Kokalj, A.; Lazzeri, M.; MartinSamos, L. et al. QUANTUM ESPRESSO: A Modular and Open-Source Software Project for Quantum Simulations of Materials. J. Phys.: Condens. Matter 2009, 21, 395502.

59. Li, W.; Carrete, J.; Katcho, N. A.; Mingo, N. ShengBTE: A Solver of the Boltzmann Transport Equation for Phonons. Comput. Phys. Commun. 2014, 185, 1747-1758.

60. Hussain, M. N.; Janajreh, I. Numerical Simulation of a Cylindrical Heat Pipe and Performance Study. Int. J. Therm. Environ. Eng. 2016, 12, 135-141. 
61. Hilty, F. W.; Tonks, M. R. Development and Application of a Microstructure Dependent Thermal Resistor Model for UO2 Reactor Fuel with High Thermal Conductivity Additives. J. Nucl. Mater. 2020, 540, 152334.

62. Sun, B.; Gu, X.; Zeng, Q.; Huang, X.; Yan, Y.; Liu, Z.; Yang, R.; Koh, Y. K. Temperature Dependence of Anisotropic Thermal-Conductivity Tensor of Bulk Black Phosphorus. Adv. Mater. 2017, 29, 1603297.

63. O’Hara, K.; Hu, X.; Cahill, D. G. Characterization of Nanostructured Metal Films by Picosecond Acoustics and Interferometry. J. Appl. Phys. 2001, 90, 4852-4858.

64. Yamamoto, Y.; Imai, T.; Tanabe, K.; Tsuno, T.; Kumazawa, Y.; Fujimori, N. The Measurement of Thermal Properties of Diamond. Diamond Relat. Mater. 1997, 6, 1057-1061.

65. Lindsay, L.; Broido, D.; Reinecke, T. Thermal Conductivity and Large Isotope Effect in GaN from First Principles. Phys. Rev. Lett. 2012, 109, 095901.

66. Touloukian, Y.; Powell, R.; Ho, C.; Klemens, P. Thermal Conductivity: Nonmetallic Solids Volume 2 of Thermophysical Properties of Matter Eds YS Touloukian. CY Ho (New York: IFI/Plenum) pp 1970, 182-193.

67. Zheng, Q.; Li, S.; Li, C.; Lv, Y.; Liu, X.; Huang, P. Y.; Broido, D. A.; Lv, B.; Cahill, D. G. High Thermal Conductivity in Isotopically Enriched Cubic Boron Phosphide. Adv. Funct. Mater. 2018, 28, 1805116.

68. Shibata, H.; Waseda, Y.; Ohta, H.; Kiyomi, K.; Shimoyama, K.; Fujito, K.; Nagaoka, H.; Kagamitani, Y.; Simura, R.; Fukuda, T. High Thermal Conductivity of Gallium Nitride (GaN) Crystals Grown by HVPE Process. Mater. Trans. 2007, 48, 2782-2786. 